\title{
Triplet Higgs boson collider phenomenology after the LHC
}

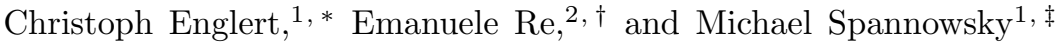 \\ ${ }^{1}$ Institute for Particle Physics Phenomenology, Department of Physics, \\ Durham University, Durham DH1 3LE, United Kingdom \\ ${ }^{2}$ Rudolf Peierls Centre for Theoretical Physics, Department of Physics, \\ University of Oxford, Oxford, OX1 3NP, United Kingdom
}

\begin{abstract}
ATLAS and CMS have discovered a Standard Model Higgs-like particle. One of the main discovery channels is the Higgs decay to two photons, which, at the moment, seems to be considerably enhanced over the Standard Model expectation. Models with additional charged matter coupling to the Higgs sector can enhance or decrease the $h \rightarrow \gamma \gamma$ branching ratio. We take this as motivation to confront the so-called Georgi-Machacek model of Higgs triplets with the results of recent searches for a SM Higgs boson performed at the LHC. We also investigate the model in regions of the allowed parameter space relevant for a SM-like phenomenology. The Georgi-Machacek model avoids tree-level issues of the $T$ parameter, while offering a vastly modified Higgs phenomenology compared to the Standard Model. This comes at the price of introducing another fine-tuning problem related to electroweak precision measurements. We investigate the collider phenomenology of the Georgi-Machacek model in the light of existing collider constraints beyond any effective field theory approximation and contextualize our findings with electroweak precision constraints.
\end{abstract}

\section{INTRODUCTION}

ATLAS and CMS have reported on the discovery of a Standard Model Higgs-like particle [1] with a mass of approximately $126 \mathrm{GeV}$ [2] [5]. Bounds on production of SM Higgs-like states with heavier masses have been established as low as $\sigma \times \mathrm{Br} /[\sigma \times \mathrm{Br}]_{\mathrm{SM}} \simeq 0.1$.

In the light of the late discovery which hints at deviations from the SM expectation, attempts have been made to reconcile the excess in $h \rightarrow \gamma \gamma$ in correlation with underproduction or "spot-on" production in the other decay and search channels. Given that there is still consistency with the SM Higgs hypothesis within 1 to 2 sigma, these results are easily misinterpreted. Preferring one model over the other on basis of a better $\chi^{2}$ fit can be misleading: Taking the tension of the Higgs mass of $126 \mathrm{GeV}$ already within the SM as an indicator of the SM's validity is certainly at odds with the tremendous success that the SM has experienced so far, culminating in the late LHC discovery.

Another way to check the validity of a certain model is to map the uncertainty of the cross sections' extraction from data onto the extended Higgs potential's parameters (and vice versa). Doing so, the SM can in principle be recovered from the Higgs sector extension for hypothetically accurate measurements without errors if the model can approach a phenomenologically well-defined decoupling limit.

An enhancement of the $h \rightarrow \gamma \gamma$ rate can typically be achieved by including additional charged states which are singlets under QCD. This predominantly alters the de-

\footnotetext{
*Electronic address: christoph.englert@durham.ac.uk

$\dagger$ Electronic address: emanuele.re@physics.ox.ac.uk

‡Electronic address: michael.spannowsky@durham.ac.uk
}

cay branching ratios while leaving the production cross sections unmodified apart from higher order corrections and mixing effects. Higgs triplet extensions which included color-neutral but up to doubly charged scalar particles are therefore well-motivated model-building options to reconcile the current observations [6] 8]. Constraints from electroweak precision measurements and the correlation of the Higgs candidate production cross section with exclusion bounds, that are relevant for the remaining Higgs particles, are typically treated as a nuisance in this context.

In simple triplet Higgs extensions, i.e. by introducing a complex $\mathbf{3}_{1}$ under $\mathrm{SU}(2)_{L} \times \mathrm{U}(1)_{Y}$, the additional Higgs bosons' phenomenology in SM-like search channels is typically suppressed. This comes from the fact that their couplings to the SM fields are controlled by unitarity requirements being saturated by the $126 \mathrm{GeV}$ candidate and by the small triplet's vacuum expectation value (vev) needed for consistency with the $T$ parameter (the $W / Z$ mass ratio) 9 . If the $T$ parameter issue is resolved at tree-level by including another real triplet Higgs field under $\mathrm{SU}(2)_{L}$, in what has become known as the GeorgiMachacek model [10], the Higgs phenomenology becomes more involved, and the current measurements imply nontrivial constraints on particle masses, couplings and the extended Higgs potential.

In the present paper we confront the Georgi-Machacek model of Higgs triplets [10] with the measured results from LHC Higgs searches of the 7 and $8 \mathrm{TeV}$ runs. More precisely, we perform scans over a simplified version of the model's potential to identify the parameter region which is allowed in the light of current direct searches and electroweak precision measurements. Doing so, our approach is complementary to previous work by other groups (see e.g. Refs. [6, 8, 11-14]). Instead of using an effective lagrangian to extract information on the Higgs 
couplings from data and then map these constraints on the model parameter space, we use the full model and compare its predictions at a given point in the parameter space with the observed data. We also include constraints from electroweak precision measurements performed during the LEP era, thus providing (to our knowledge) the most detailed analysis of this model in the context of LEP and LHC collider measurements hitherto.

This work is organized as follows. We review the Georgi-Machacek (GM) model in Sec. II to make this paper self-contained and comprehensive. In particular we introduce the potential that we scan in the remainder of this work. In Sec. III we discuss the bounds which we take into account when scanning over the extended Higgs sector phenomenology. We also give some technical details of our implementations. Sec. IV is finally devoted to results, where we also detail the parameter choices of our scan. We present our conclusions in Sec. V.

\section{THE MODEL}

It is well-known that Higgs triplets naively face compatibility issues with electroweak precision data. This is due to the fact that a simple triplet Higgs-extension of the Higgs potential leads to tree-level custodial isospin violation, which is not present for complex (symplectic) $\mathrm{SU}(2)_{L}$ doublets accidentally (as a consequence of renormalizability and gauge invariance). This violation requires the Higgs triplet's vev to be small compared to the weak scale in order to obtain the experimentally observed $m_{W} / m_{Z}$ mass ratio.

Reconciling the $\rho=1+\alpha T$ parameter (at least at tree-level) in a model with triplets requires more than a single triplet field [10]. This can be seen by reminding ourselves of how custodial isospin comes about for the SM doublet $\Phi$ : If $\Phi$ transforms as a $\mathbf{2}$ under $\mathrm{SU}(2)_{L}$, then so does $\Phi^{c}=i \sigma^{2} \Phi^{*}$. Consequently, the Higgs potential depending only on $|\Phi|^{2}$ has a larger symmetry $\mathrm{SU}(2)_{L} \times \mathrm{SU}(2)_{R} \simeq \mathrm{SO}(4)$, which breaks to $\mathrm{SU}(2)_{D}$ after the Higgs obtains its vev. This ensures that the resulting electroweak gauge boson masses are related by only the weak mixing angle. In order to establish a $\mathrm{SU}(2)_{R}$ global symmetry in the Higgs potential also in presence of $\mathrm{SU}(2)_{L}$ triplets, we need to enlarge the field content with a real triplet such that $\mathrm{SU}(2)_{R}$ can act on the complex triplet, its charge-conjugated version and the real triplet.

The GM model therefore introduces the Higgs fields

$$
\Phi=\left(\begin{array}{cc}
\phi_{2}^{*} & \phi_{1} \\
-\phi_{1}^{*} & \phi_{2}
\end{array}\right), \quad \Xi=\left(\begin{array}{ccc}
\chi_{3}^{*} & \xi_{1} & \chi_{1} \\
-\chi_{2}^{*} & \xi_{2} & \chi_{2} \\
\chi_{1}^{*} & -\xi_{1}^{*} & \chi_{3}
\end{array}\right)
$$

In this notation, $\Phi$ is simply a SM-like Higgs doublet and $\Xi$ combines the complex $\left(\chi_{1}, \chi_{2}, \chi_{3}\right)$ and real $\left(\xi_{1}, \xi_{2},-\xi_{1}^{*}\right)$ triplets. Note that while symmetry breaking with a correct $\rho$ parameter can be fully achieved with only $\Xi$, the introduction of fermion mass terms still requires the presence of a SM-like Higgs doublet.

The Higgs sector lagrangian that we consider in the remainder is

$$
\begin{aligned}
\mathcal{L}=\frac{1}{2} \operatorname{Tr}\left[D_{2, \mu} \Phi^{\dagger} D_{2}^{\mu} \Phi\right] & +\frac{1}{2} \operatorname{Tr}\left[D_{3, \mu} \Xi^{\dagger} D_{3}^{\mu} \Xi\right]-V(\Phi, \Xi) \\
& +\Phi \text { Yukawa interactions , }
\end{aligned}
$$

with the potential

$$
\begin{gathered}
V(\Phi, \Xi)=\frac{\mu_{2}^{2}}{2} \operatorname{Tr}\left(\Phi^{c} \Phi\right)+\frac{\mu_{3}^{2}}{2} \operatorname{Tr}\left(\Xi^{c} \Xi\right)+\lambda_{1}\left[\operatorname{Tr}\left(\Phi^{c} \Phi\right)\right]^{2} \\
+\lambda_{2} \operatorname{Tr}\left(\Phi^{c} \Phi\right) \operatorname{Tr}\left(\Xi^{c} \Xi\right)+\lambda_{3} \operatorname{Tr}\left(\Xi^{c} \Xi^{c} \Xi\right) \\
+\lambda_{4}\left[\operatorname{Tr}\left(\Xi^{c} \Xi\right)\right]^{2}-\lambda_{5} \operatorname{Tr}\left(\Phi^{c} t_{2}^{a} \Phi t_{2}^{b}\right) \operatorname{Tr}\left(\Xi^{c} t_{3}^{a} \Xi t_{3}^{b}\right) . \quad(2 \mathrm{~b})
\end{gathered}
$$

$D_{2}, D_{3}$ are the covariant derivatives for the doublet and triplet representations, respectively, e.g.

$$
D_{2, \mu} \Phi=\partial_{\mu} \Phi+i g_{w} t_{2}^{a} W_{\mu}^{a} \Phi-i g_{y} B_{\mu} \Phi t_{2}^{3}
$$

Hypercharge $\mathrm{U}(1)_{Y}$ is embedded into $\mathrm{SU}(2)_{R}$ as in the SM. The $\mathfrak{s u}(2)$ generators are $t_{2}^{a}=\sigma^{a} / 2$ and

$$
\begin{gathered}
t_{3}^{1}=\frac{1}{\sqrt{2}}\left(\begin{array}{lll}
0 & 1 & 0 \\
1 & 0 & 1 \\
0 & 1 & 0
\end{array}\right), \quad t_{3}^{2}=\frac{i}{\sqrt{2}}\left(\begin{array}{ccc}
0 & -1 & 0 \\
1 & 0 & -1 \\
0 & 1 & 0
\end{array}\right) \\
t_{3}^{3}=\left(\begin{array}{ccc}
1 & 0 & 0 \\
0 & 0 & 0 \\
0 & 0 & -1
\end{array}\right)
\end{gathered}
$$

The potential in Eq. 2b is a simplified version of the allowed terms documented in Refs. [15, 16]: the more general renormalizable and gauge invariant potential would allow different vevs for the 2 triplet fields, and would also include cubic terms (Eq. (B3) of Ref. [15]). Terms of the former type are $\mathrm{SU}(2)_{R^{-v}}$ violating, and we avoid them by requiring exact custodial invariance at tree level, as remarked in the following. Terms with an odd number of scalars can be easily avoided by means of a $\mathbb{Z}_{2}$ symmetry acting onto the triplet fields. Moreover our analysis is only indirectly affected by the Higgs trilinear couplings (for measurement strategies of the latter see Ref. [17]), and therefore our results are general enough to assess the impact of Higgs measurements. Hence, our choice for $V(\Phi, \Xi)$ should be thought of as a minimal ansatz, that captures the important features of Higgs triplet phenomenology such as modified Higgs branching ratios, production cross sections and Higgs mixing in a well-defined way.

Switching off hypercharge gauging and the Yukawa interactions, the lagrangian is manifestly invariant under $\mathrm{SU}(2)_{L} \times \mathrm{SU}(2)_{R}$. Gauging a subgroup amounts to explicit breaking of custodial isospin, and the effects of custodial isospin violation are steered by gauge and Yukawa couplings. Hence a small $T$ parameter can be considered natural [18. 
Electroweak symmetry breaking is triggered by the scalar fields developing vevs

$$
\langle\Phi\rangle=v_{\Phi} / \sqrt{2} \mathbb{1}, \quad\langle\Xi\rangle=v_{\Xi} \mathbb{1},
$$

as a consequence of minimizing the Higgs potential which also allows us to eliminate $\mu_{2,3}^{2}$ and express them as functions of vevs and $\lambda$ 's. The vevs in Eq. (5) are in accordance with preserved custodial isospin. In principle we could have $\left\langle\chi_{3}\right\rangle \neq\left\langle\xi_{2}\right\rangle$, which would be induced in a $\mathrm{SU}(2)_{R}$-violating potential. In the following we impose $\mathrm{SU}(2)_{R}$ invariance and such terms are absent. This will have interesting consequences for the $T$ parameter.

The masses of the electroweak bosons $m_{W}, m_{Z}$ after symmetry breaking follow the usual pattern of vev $\times$ electroweak coupling, but the electroweak scale is now generated by both the doublet and the triplet vevs

$$
(246 \mathrm{GeV})^{2} \simeq v_{\mathrm{SM}}^{2}=v_{\Phi}^{2}+8 v_{\Xi}^{2} .
$$

As usual, it is useful to parametrize the relative contribution of $v_{\Phi}$ and $v_{\Xi}$ to $v_{\mathrm{SM}}$ via trigonometric functions:

$$
\begin{aligned}
& \cos \theta_{H}=: c_{H}=\frac{v_{\Phi}}{v_{\mathrm{SM}}}, \\
& \sin \theta_{H}=: s_{H}=\frac{2 \sqrt{2} v_{\Xi}}{v_{\mathrm{SM}}} .
\end{aligned}
$$

Compared to "ordinary" complex triplet Higgs extensions, as considered recently in, e.g., Ref. [7, 19] to reconcile the $h \rightarrow \gamma \gamma$ enhancement, there is no requirement to have a hierarchy $v_{\Xi} \ll v_{\Phi}$, i.e. the phenomenology of electroweak bosons can be highly different from the SM without being in immediate tree-level conflict with electroweak precision data. This is our main motivation to re-interpret the current Higgs results in the context of the GM model.

The masses that arise from expanding the extended Higgs sector around the minimum can be classified according to custodial isospin following the above remarks. Eq. 22 yields a quintet, two triplets and two singlets. The massless triplet contains the longitudinal degrees of freedom of the electroweak gauge bosons, while the singlets mix as a consequence of Eq. (2b). After diagonalizing the singlet mixing of $H_{\Phi}, H_{\Xi^{\ddagger}}^{\ddagger}$

$$
\begin{aligned}
& H_{0}=c_{q} H_{\Phi}+s_{q} H_{\Xi}, \\
& H_{0}^{\prime}=-s_{q} H_{\Phi}+c_{q} H_{\Xi},
\end{aligned}
$$

the Higgs mass spectrum reads

$$
\begin{aligned}
& m_{H_{0}}^{2}=2\left(2 \lambda_{1} v_{\Phi}^{2}+2\left(\lambda_{3}+3 \lambda_{4}\right) v_{\Xi}^{2}+m_{\Phi \Xi}^{2}\right), \\
& m_{H_{0}^{\prime}}^{2}=2\left(2 \lambda_{1} v_{\Phi}^{2}+2\left(\lambda_{3}+3 \lambda_{4}\right) v_{\Xi}^{2}-m_{\Phi \Xi}^{2}\right), \\
& m_{H_{3}}^{2}=\frac{1}{2} \lambda_{5}\left(v_{\Phi}^{2}+8 v_{\Xi}^{2}\right), \\
& m_{H_{5}}^{2}=\frac{3}{2} \lambda_{5} v_{\Phi}^{2}+8 \lambda_{3} v_{\Xi}^{2},
\end{aligned}
$$

\footnotetext{
$\ddagger H_{\Phi} \sim \Re\left\{\phi_{2}\right\}$ whereas $H_{\Xi}$ is the linear combination of $\xi_{2}$ and $\Re\left\{\chi_{3}\right\}$ which yields a custodial singlet.
}

where

$$
\begin{aligned}
& m_{\Phi \Xi}^{2}=\left[4 \lambda_{1}^{2} v_{\Phi}^{4}-8 \lambda_{1}\left(\lambda_{3}+3 \lambda_{4}\right) v_{\Phi}^{2} v_{\Xi}^{2}\right. \\
& \left.\quad+v_{\Xi}^{2}\left(3\left(2 \lambda_{2}-\lambda_{5}\right)^{2} v_{\Phi}^{2}+4\left(\lambda_{3}+3 \lambda_{4}\right)^{2} v_{\Xi}^{2}\right)\right]^{1 / 2},
\end{aligned}
$$

and

$$
\begin{aligned}
\sin \angle\left(H_{\Phi},\right. & \left.H_{0}\right)=: s_{q} \\
= & \frac{\sqrt{3}}{\sqrt{3+\left[\frac{2 \lambda_{1} v_{\Phi}^{2}-2\left(\lambda_{3}+3 \lambda_{4}\right) v_{\Xi}^{2}+m_{\Phi \Xi}^{2}}{\left(2 \lambda_{2}-\lambda_{5}\right) v_{\Phi} v_{\Xi}}\right]^{2}}} .
\end{aligned}
$$

Note that the model contains a $\mathcal{C P}$ odd scalar $H_{3}^{0}$ which is the neutral component of the massive custodial triplet (more precisely, the $\mathcal{C P}$ odd scalar is the non- $Z_{L}$ combination of $\Im\left\{\phi_{2}\right\}$ and $\Im\left\{\chi_{3}\right\}$ ).

Writing the full lagrangian in terms of the mass eigenstates we recover the Feynman rules of the theory. For a general gauge (i.e. including the Goldstone sector) we find $\mathcal{O}(500)$ potentially non-zero interaction vertices obviously too many to discuss here. Most of the Feynman rules, however, arise from the Higgs sector including the Higgs self couplings, Eq. (2b). We have computed the Feynman rules using FeynRules [20] and validated the output against an in-house Feynman rule extraction code. Some of the Feynman rules can be found in earlier publications 6, 11, 12, 15, 16, 21 and we also have performed analytical comparisons with these results ${ }^{\S}$.

Before we discuss the bounds which we take into account for the results of Sec. IV let us foreclose some characteristic properties of the GM model Eq. (1)-11):

1.) In contrast to models with a doublet and one complex triplet only, the GM model a priori allows for sizeable triplet vevs. Therefore, the couplings of the neutral Higgs mass eigenstates (in particular the two custodial singlets $H_{0}$ and $H_{0}^{\prime}$ ) can highly differ from the SM Higgs couplings. We denote the universal ratios of the $h V V$ and $h f \bar{f}$ couplings of $H_{0}, H_{0}^{\prime}$ with respect to the SM values by

$$
\begin{aligned}
c_{t, H_{0}} & =\frac{c_{q}}{c_{H}}, \\
c_{v, H_{0}} & =c_{q} c_{H}+\sqrt{8 / 3} s_{q} s_{H}, \\
c_{t, H_{0}^{\prime}} & =-\frac{s_{q}}{c_{H}}, \\
c_{v, H_{0}^{\prime}} & =-s_{q} c_{H}+\sqrt{8 / 3} c_{q} s_{H} .
\end{aligned}
$$

\footnotetext{
$\S$ In particular, to recover the Feynman rules for the Higgs self couplings listed in [16], we notice that the $\lambda$ 's couplings of [16] can be obtained from ours with the substitutions $\lambda_{1} \rightarrow \lambda_{1}+\lambda_{3}, \lambda_{2} \rightarrow$ $2 \lambda_{3}+\lambda_{4}, \lambda_{3} \rightarrow 3 \lambda_{5}, \lambda_{4} \rightarrow \lambda_{2}+\lambda_{3}-\lambda_{5}, \lambda_{5} \rightarrow 2 \lambda_{4}$. A number of Feynman rules in the GM phenomenology-pioneering work of Ref. [16] have been superseded in Ref. [6].
} 
The above equations show that $c_{v}$ can be larger than unity, a typical property of models with weak isospin $j>1 / 2$ multiplets 22 . It is interesting to observe from the above expressions that values $\left|c_{v}\right|>1$ are possible only when the term with the prefactor $\sqrt{8 / 3} \sim 1.6$ dominates over the first term. Values of $\left|c_{v}\right|$ very close to one can be obtained either when the first term is very close to unity (which means very small triplet vev and minimal mixing in the singlet sector, i.e. $c_{q}$ very close to one or zero) or when there is a compensation between the two terms in $\left|c_{v}\right|$. This compensation can potentially take place only when the second term is not too suppressed, i.e. an enhanced $\left|c_{v}\right|$ does not occur for $0 \leq s_{H} \ll 1$.

2.) Another very interesting feature of the GM model shows up for vanishing mixing of the singlet states In this situation the mass eigenstates are by definition aligned with the $H_{\Phi}, H_{\Xi}$ states. If we take the limit $s_{H}\left(c_{H}\right) \simeq 0$, which corresponds to breaking electroweak symmetry mainly with the doublet (triplet) vev, we get a massless scalar in the spectrum. This state always corresponds to $H_{0}^{\prime}$ and, as we will see, it has important consequences for the model's phenomenology.

Away from the edges of the parameter space (i.e. when neither $s_{H}$ nor $c_{H}$ are very small, and $\lambda$ 's are all of the same order), the above mechanism can be rephrased by saying that the lighter custodial singlet Higgs boson is usually the one which is less related to electroweak symmetry breaking [16]. When the mixing in the singlets sector is non-negligible, the mass spectrum becomes effectively less affected by this property, and the mass of the lightest Higgs bo- son can be made sizeable: the allowed size of this misalignment is encoded in our results.

Moreover, despite of these considerations, all states clearly contribute to unitarization of longitudinal gauge boson scattering (the longitudinal gauge bosons are mixtures of the triplet $\Xi$ and doublet $\Phi)$, and this has interesting implications for the electroweak precision constraints, especially in the scenario discussed in Sec. IVA.

For a more general potential that also violates $\mathrm{SU}(2)_{R}$ invariance, the $s_{H} \rightarrow 0$ limit does not necessarily involve $m_{H_{0}^{\prime}} \rightarrow 0$, cf. [15]. For these more general potentials there always exist parameter choices that involve light scalars in the spectrum. The correlation with $s_{H}$, however, is not so obvious. Generically the light scalars follow from parameters with a small $\mathrm{SU}(2)_{R}$ breaking. Since $\rho \simeq 1$ is well-established experimentally, invariance under global $\mathrm{SU}(2)_{R}$ is wellestablished too, and the allowed isospin violation can be treated as a perturbation which should restore, to large extent, the correlation between vanishing $s_{H}$ and $m_{H_{0}^{\prime}} \rightarrow 0$, for phenomenologically allowed choices. In fact, the limit $v_{\Xi} \rightarrow 0$ in Eq. (2b) is tantamount to $\mu_{3}^{2} \rightarrow\left(-2 \lambda_{2}+\lambda_{5}\right) v_{\mathrm{SM}}^{2}$, which means that the custodial singlet part of the Higgs potential effectively "sees" a vanishing mass term, while this is not the case for the custodial triplet and quintet.

\section{BOUNDS}

The implications of the GM model for the PeskinTakeuchi $S, T, U$ parameters [9],

$$
\begin{aligned}
& S=\frac{4 s_{w}^{2} c_{w}^{2}}{\alpha}\left(\frac{\Pi_{Z Z}\left(m_{Z}^{2}\right)-\Pi_{Z Z}(0)}{m_{Z}^{2}}-\frac{c_{w}^{2}-s_{w}^{2}}{c_{w} s_{w}} \frac{\Pi_{A Z}\left(m_{Z}^{2}\right)-\Pi_{A Z}(0)}{m_{Z}^{2}}-\frac{\Pi_{A A}\left(m_{Z}^{2}\right)-\Pi_{A A}(0)}{m_{Z}^{2}}\right), \\
& T=\frac{1}{\alpha}\left(\frac{\Pi_{W W}(0)}{m_{W}^{2}}-\frac{\Pi_{Z Z}(0)}{m_{Z}^{2}}-\frac{2 s_{w}}{c_{w}} \frac{\Pi_{A Z}(0)}{m_{z}^{2}}-\frac{s_{w}^{2}}{c_{w}^{2}} \frac{\Pi_{A A}(0)}{m_{Z}^{2}}\right), \\
& U=\frac{4 s_{w}^{2}}{\alpha}\left(\frac{\Pi_{W W}\left(m_{W}^{2}\right)-\Pi_{W W}(0)}{m_{W}^{2}}-c_{w}^{2} \frac{\Pi_{Z Z}\left(m_{Z}^{2}\right)-\Pi_{Z Z}(0)}{m_{Z}^{2}}\right. \\
& \left.-s_{w}^{2} \frac{\Pi_{A A}\left(m_{Z}^{2}\right)-\Pi_{A A}(0)}{m_{Z}^{2}}-2 s_{w} c_{w} \frac{\Pi_{A Z}\left(m_{Z}^{2}\right)-\Pi_{A Z}(0)}{m_{Z}^{2}}\right),
\end{aligned}
$$

have been pioneered in Ref. [23.

The parameters $\Pi_{X Y}$ denote the gauge boson polarization functions which are obtained from the two-point functions $X \rightarrow Y(X, Y=A, Z, W)$,

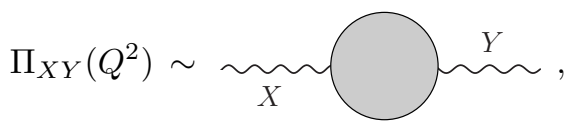

$\alpha$ is the fine structure constant and $s_{w}, c_{w}$ are the sine and cosine of the weak mixing angle as usual. The blob denotes all particle dynamics which enters the polarization functions in the corresponding model.

At this stage, it is important to note a peculiarity of the $T$ parameter that arises in the tree-level $\mathrm{SU}(2)_{R}$ symmetric GM model. Although the identification between the real and complex triplets' vevs yields the cor- 
rect tree-level $W / Z$ mass ratio, the situation becomes more involved at the one-loop. The real triplet's vev requires the introduction of a counter term (related to the weak mixing angle renormalization) that could be naively unexpected from the $\mathrm{SU}(2)_{R}$-symmetric treelevel lagrangian 23. This counter-term mends a residual UV singularity in the $T$ parameter. In this sense, $\mathrm{SU}(2)_{R}$ invariance introduces an additional naturalness problem in addition to the conventional hierarchy problem, namely why the $T$ parameter remains small. This finding is somewhat similar to the original $T$ parameter problem in $\mathrm{SU}(2)_{R}$-violating triplet extensions.

Performing the full one-loop calculation with the Feynman rules described in the previous section, we find that, after introducing a corresponding $T$ parameter counter term, our calculation is manifestly free of UV divergencies; no residual UV divergencies appear in neither the $S$ nor in the $U$ parameter, in accordance with the results of Ref. [23].

As pointed out in Ref. 23 the GM model picks up a quadratic $T$ parameter divergence which introduces a fine-tuning problem analogous to the Higgs mass. The $T$ parameter constraint is very stringent and will probe the finite logarithms. If these logarithms are of order one, a large one-loop $T$ parameter can decrease again when the full perturbative expansion is known $\%$. We therefore trace the effect of imposing $S$ independent from $T$ in Sec. IV. When imposing only the $S$ parameter constraint we set $T=0$. This can in principle be interpreted as a situation where a large $T$ shows up as relic of perturbation theory.

At this point it is worthwhile to comment on the applicability of $S, T, U$ for the present model. Estimating new physics effects by means of $S, T, U$ assumes, among other approximations 25], that the new physics scale $\Lambda$ is larger than $m_{Z}$. Identifying $m_{H_{0}}$ as the $126 \mathrm{GeV}$ candidate we can face spectra for which $m_{H_{0}^{\prime}}$ is close to or even below the $Z$ threshold. In this case, the expansion of the vacuum polarization functions in terms of $Q^{2} / \Lambda^{2}$ on which $S, T, U$ rely** could be ill-defined if the coupling $\left|c_{v, H_{0}^{\prime}}\right|$ is large. Then, the light scalar cannot be considered a small perturbation compared to the heavier states' impact. Including the LEP constraints on light SM-like Higgs production $\left(m_{H} \leq 114 \mathrm{GeV}\right)$, which probe combined mixings down to $c_{v}^{2} \sim 0.01$, the $S, T, U$ bounds are superseded by the direct exclusion from $e^{+} e^{-} \rightarrow h Z$ searches. If the mixing is too large, the direct LEP constraints remove the parameter point from the list of valid points, especially when the resulting $\Delta S$ falls into the al-

I See e.g. Ref. 24] for a solution to the Higgs naturalness problem in this context

** We would like to remind the reader that small allowed $S, T, U$ values are necessary but not sufficient conditions for consistency with electroweak precision data. The latter requires carrying out an analysis including model-dependent radiative corrections to at least next-to leading order. lowed region, and our theoretical predicament is resolved phenomenologically.

Additional constraints that in principle reduce the model's allowed parameter space follow from non-oblique corrections, e.g. corrections the $Z b \bar{b}$ vertex, and flavor physics. Modifications of the flavor sector are induced by "rotating in" the custodial triplet $\left(\mathrm{H}_{3}^{+}, \mathrm{H}_{3}^{0}, \mathrm{H}_{3}^{-}\right)$via Goldstone mixings in the extended space of Higgs fields, Eq. (1), and by the singlets' mixing.

These mixings are parametrically controlled by $c_{q}, s_{q}, c_{H}$ and $s_{H}$. The qualitative modifications in the flavor sector in comparison to the SM are similar to a two-Higgs doublet model without flavor changing neutral currents [26]. We expect that consistency with flavor constraints can be achieved without too large quantitative changes induced (see e.g. Ref. [8]). Especially for scenarios with tuned $T$, which, as we will see in Sec. [IV] are consistent for small $s_{H}$, we can expect flavor physics to be SM-like. Similar arguments hold for the non-oblique corrections, e.g. the $Z b \bar{b}$ vertex does not receive corrections from the quintet states at the one-loop level [27.

Beyond indirect constraints such as electroweak precision measurements, there are already direct constraints from searches $H_{5}^{ \pm \pm} \rightarrow \ell^{ \pm} \ell^{ \pm}(\ell=e, \mu)$ by both ATLAS and CMS 28. Such a decay can be prompt by the introduction of a Majorana-type interaction term of the triplet and the leptons and constrains a different sector of the model. The bounds of Ref. [28] assume coupling strengths of order 1 to derive lower mass limits on the quintet mass. While this assumption is reasonable to obtain a well-defined limit in hypothesis tests, especially for a heavy quintet $m_{H_{5}} \gtrsim 2 m_{W}$ this is not reasonable. For large $m_{H_{5}}$, we need to include a partial decay width which is dominated by longitudinally polarized $W$ bosons. This leads to a tree-level dependence of the partial decay width $\Gamma\left(H_{5}^{ \pm \pm} \rightarrow W^{ \pm} W^{ \pm}\right) \sim m_{H_{5}}^{3} / m_{W}^{2}$. Just like in the SM, the decay to light fermions scales $\Gamma\left(H_{5}^{ \pm \pm} \rightarrow \ell^{ \pm} \ell^{ \pm}\right) \sim m_{H_{5}}$, and the decay to same-sign leptons quickly becomes subdominant. Searches for samesign inclusive dileptons exist [29] but are typically designed to cope with non-resonant squark-gluino production [29, 30]. Additional constraints from leptonic final states can be obtained from measurements of lepton flavor violating $\mu^{ \pm} \rightarrow e^{+} e^{-} e^{ \pm}[31333$.

We do not include these constraints in detail in the following. We however note that dedicated limits can be obtained from an analysis of the $\ell^{ \pm} \ell^{ \pm} j j$ final state for $H_{5}^{ \pm \pm}$production via Weak Boson Fusion (WBF).

\section{RESULTS}

We now turn to the results of our scans over the Higgs potential. The role of the Higgs candidate can in principle be played by all uncharged Higgs states $H_{0}, H_{0}^{\prime}, H_{3}^{0}, H_{5}^{0}$. In the light of recent results, however, it is unreasonable to study $H_{3}^{0}$ and $H_{5}^{0}$ in further detail:

- $H_{3}^{0}$ is a $\mathcal{C P}$ odd state which does not couple to 
the massive vector bosons. The measurement of the Higgs candidate in the $Z Z$ channel [2, 3] which also disfavors pure $\mathcal{C P}$ odd quantum numbers from angular distributions in the leptonic decay channels 34 removes $H_{3}^{0}$ from our list of candidates. Note that constraints on $\mathcal{C P}$ odd scalars directly constrain $\lambda_{5}$, Eq. (9). Furthermore, a $126 \mathrm{GeV}$ $H_{3}^{0}$ is also disfavored from flavor physics and nonoblique corrections [27.

- Even though $H_{5}^{0}$ has the right quantum numbers and vertex structures to mimic the branchings to the $\gamma \gamma, Z Z$ and $W W$ final states, the quintet is fermiophobic as a consequence of the $\mathrm{SU}(2)_{R}$ symmetry. This removes gluon fusion [35] as production mode and leaves inclusive weak boson fusion 36 as the largest production cross section for $H_{5}^{0}$. Weak boson fusion for inclusive cuts is typically suppressed by one order of magnitude compared to gluon fusion [37]. Reconciling the measurements in $Z Z, W W, \gamma \gamma, \tau^{+} \tau^{-}$with the observed rates is therefore impossible, and $H_{5}^{0}$ cannot play the role of the $126 \mathrm{GeV}$ candidate, when considering recent limits on the total Higgs width [38].

From Eq. (9) it is manifest that $H_{0}^{\prime}$ is always lighter than $H_{0}$, and hence $H_{0}^{\prime}$ is the natural candidate to play the role of the observed Higgs. As already mentioned in Sec. III, we will also study the scenario where there is a lighter uncharged scalar state in the mass spectrum, which we can achieve by fixing $m_{H_{0}}=126 \mathrm{GeV}$. However we will see that this scenario is disfavored.

Although partial motivation of this work is to explore the GM model's potential to enhance the $h \rightarrow \gamma \gamma$ branching ratio via the presence of extra matter, it is clear from the Higgs couplings not fixed to the SM values (Eq. (12) that the $\gamma \gamma$ branching ratio in this model is also affected by these modified tree-level couplings. This means that it is indeed possible to find regions in the parameter space where $\operatorname{Br}(H \rightarrow \gamma \gamma)$ is very close to the SM also without forcing the charged higgses to be very heavy. We will briefly explore this possibility too, by looking for points that reproduce the Higgs signal and at the same time have a $\gamma \gamma$ branching ratio close to unity. Precise fits on Higgs couplings will eventually tell whether this is a feasible scenario, although recent studies by several collaborations favor regions with $\left|c_{t}\right|,\left|c_{v}\right| \sim 1$.

The cross section limits for the uncharged Higgs fields are adopted from the most recent LHC measurements [4, 5] and from the combined LEP constraints [39]. In particular for Higgs masses smaller then the LEP direct bound, we use the LEP bound on the $H Z Z$ coupling.

Signal strengths are defined as follows:

$$
\mu_{h \rightarrow X X}=\frac{\sigma(p p \rightarrow h) \times \operatorname{Br}(h \rightarrow X X)}{\sigma_{\mathrm{SM}}(p p \rightarrow h) \times \operatorname{Br}_{\mathrm{SM}}(h \rightarrow X X)},
$$

and for this paper we define the combined signal strength as

$$
\mu_{h}=\frac{\sigma(p p \rightarrow h) \times[\operatorname{Br}(h \rightarrow W W)+\operatorname{Br}(h \rightarrow Z Z)+\operatorname{Br}(h \rightarrow \gamma \gamma)]}{\sigma_{\mathrm{SM}}(p p \rightarrow h) \times\left[\operatorname{Br}_{\mathrm{SM}}(h \rightarrow W W)+\operatorname{Br}_{\mathrm{SM}}(h \rightarrow Z Z)+\operatorname{Br}_{\mathrm{SM}}(h \rightarrow \gamma \gamma)\right]}
$$

since ATLAS and CMS use the categories $W W, Z Z, \gamma \gamma$, $\tau \tau$ and $V b \bar{b}$ to obtain the exclusion bounds from data, and the latter two categories give a marginal contribution to $\mu_{h}$, because of non-observation of the Higgs and large experimental errors in those channels. Eq. (16) does not take into account the different channels' sensitivity which is beyond the scope of this work.

We scan $\lambda_{i} \in[-4 \pi, 4 \pi], i=1, \ldots, 5$ and $s_{H} \in[0,1]$, which also implies that since $\lambda_{5}$ is bounded from above, in our scan the maximum value allowed for $m_{H_{3}}$ is $\sim 600 \mathrm{GeV}$. To generate parameter points with $m_{H_{0}^{\prime}}\left(m_{H_{0}}\right)=126 \pm 1 \mathrm{GeV}$ more efficiently, we first generate the mass of the $126 \mathrm{GeV}$ state, and then compute $\lambda_{1}\left(\lambda_{1}\right.$ and $\left.\lambda_{2}\right)$ using Eq. (9). The generated parameter points are, hence, not flat and should by no means be understood as probability distributions of parameter points that pass the requirements.
All the LHC measurements of the Higgs-candidate properties, in particular from the $W W, Z Z$ and $\gamma \gamma$ categories, point towards an Higgs phenomenology with $\left|c_{t}\right|$ and $\left|c_{v}\right|$ not dramatically different from the SM values [8, 40, 41. Moreover, the combined signal strengths from CMS and ATLAS are $\mu_{h, \mathrm{CMS}} \simeq 0.9 \pm 0.2[5$ ] and $\mu_{h, \text { ATLAS }} \simeq 1.3 \pm 0.3$ [4]. We will take the ATLAS measurement as the paradigm of an enhanced $h \rightarrow \gamma \gamma$ production rate, but will also comment on the model's capability to reproduce the consistency with the SM as observed by CMS.

Since the theoretical expectation for these values is dominated by $\mu_{h \rightarrow W W} \sim\left|c_{t} c_{v}\right|^{2}$, it is reasonable to restrict our scans to the regions where both $\left|c_{t}\right|$ and $\left|c_{v}\right|$ are 

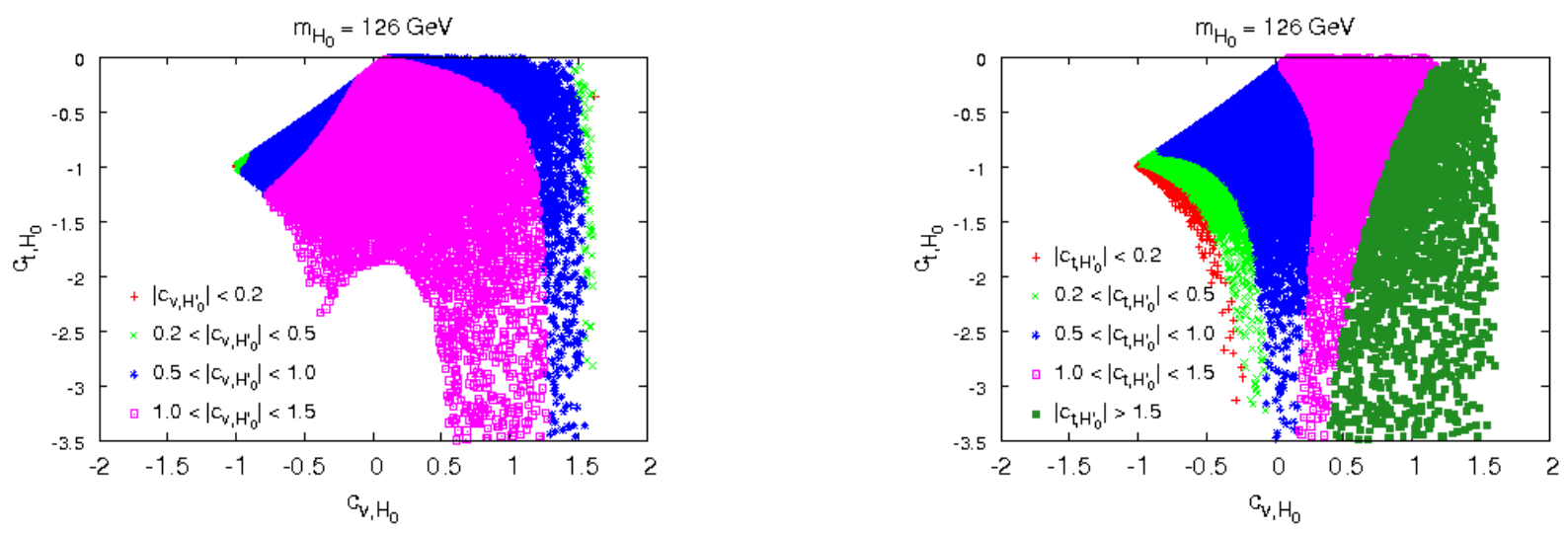

FIG. 1: $\left(c_{v, H_{0}}, c_{t, H_{0}}\right)$ correlation for $m_{H_{0}} \simeq 126 \mathrm{GeV}$. We do not impose any other additional constraint.

larger than $0.8 .^{\dagger \dagger}$. Unless otherwise stated, all results have been obtained with the aforementioned condition explicitly imposed.

We use the exclusion contours by ATLAS ${ }^{\ddagger \ddagger} 2$. In order to reproduce the observed LHC discovery signal we need an excess of $\sim 25 \%$ for the total signal strength compared to the SM hypothesis. We therefore typically need either $\left|c_{t}\right|$ or $\left|c_{v}\right|>1$. The cross section in the discovery channels scales as $c_{t}^{2}$ and the $V V$ branching ratios as $\sim c_{v}^{2} / c_{t}^{2}$. Therefore $c_{t}$ is typically less constrained in our scan, also because no statistically significant observation in the fermionic channels has been made so far. The approximate scaling for the $V V$ branching ratio mentioned above follows from the observed "SM-likeness" of the 126 $\mathrm{GeV}$ Higgs boson, which in turn implies the decay to bottom quarks to be the dominant contribution to the total decay width. Notice however that in our approach we have taken into account all the possible changes to the Higgses' total widths due to non SM-like couplings and to the extended Higgs sector. In the scenario with an uncharged scalar lighter than the $126 \mathrm{GeV}$ state, a too large deviation of the total width when $H_{0} \rightarrow H_{0}^{\prime} H_{0}^{\prime}$ opens up is limited by the experimental observation of the individual signal strengths being in good agreement with the SM values.

To estimate the signal strengths for $H_{0}, H_{0}^{\prime}$ and $H_{3}^{0}$ and compare with the exclusion bounds, we need their production cross sections and branching ratios. The branching ratios and total widths have been obtained interfacing FeynRules with a modified version BRIDGe [42, where we included the loop functions needed to evaluate the amplitudes $h \rightarrow \gamma \gamma$ via scalar, fermionic and vecto-

$\dagger \dagger$ The $\gamma \gamma$ excess, although phenomenologically very important if confirmed, cannot be responsible for a $20 \%$ enhancement of the global $\mu_{h}$ value, being a potentially large effect in a rare branching ratio.

$¥ \ddagger$ While the exclusion limits of both ATLAS and CMS do not coincide at face value, they quantitatively follow the same pattern. rial loops. We have also included the more important QCD corrections to the $h \rightarrow b \bar{b}, h \rightarrow c \bar{c}$ and $h \rightarrow g g$ decays [43, and cross-checked the implementation against the SM partial widths quoted in [37]. BRIDGE also computes the branching ratios for off-shell decays.

The leading-order inclusive cross sections $\sigma(g g \rightarrow h)$ have been computed using an adapted version of the PowheG-Box program 44. Using LO cross sections is a good approximation since higher order QCD corrections play a minor role as far as signal strengths are concerned. In doing so, we have also neglected the contribution from Higgs production via WBF, which plays a subleading role in our study. We leave a detailed discussion of the GM's WBF phenomenology to the future.

To impose our electroweak precision criteria we use the $S, T,(U \equiv 0)$ fits at $95 \%$ confidence level of Ref. 45$)^{\S \S}$. We classify a point in the parameter space as "good" with respect to the $m_{H_{0}}, m_{H_{0}^{\prime}} \simeq 126 \mathrm{GeV}$ triplet hypotheses according to three main different requirements as already alluded to before.

(i) loose electroweak precision: $S$ within the $95 \% \mathrm{CL}$ ellipse, no constraint on $T$. We understand this case as $T \equiv 0$, along the lines of Sec. III.

(ii) enforced electroweak precision: Both $S, T$ strictly in the $95 \%$ CL ellipse.

(iii) bounds from global direct searches and tagged categories: in this case we reproduce the $\mu_{h}$ value for the Higgs signal within the quoted error of $\simeq 25 \%$ and we do not violate the exclusion bounds for the other neutral Higgses. We also reproduce $\mu_{h \rightarrow W W}$ and $\mu_{h \rightarrow \gamma \gamma}$ within the $1 \sigma$ error band.

$\S \S$ During the course of this work, these fits have been updated including the Higgs measurements [46]. The differences compared to Ref. 45] are quantitatively small and not relevant for our results. 

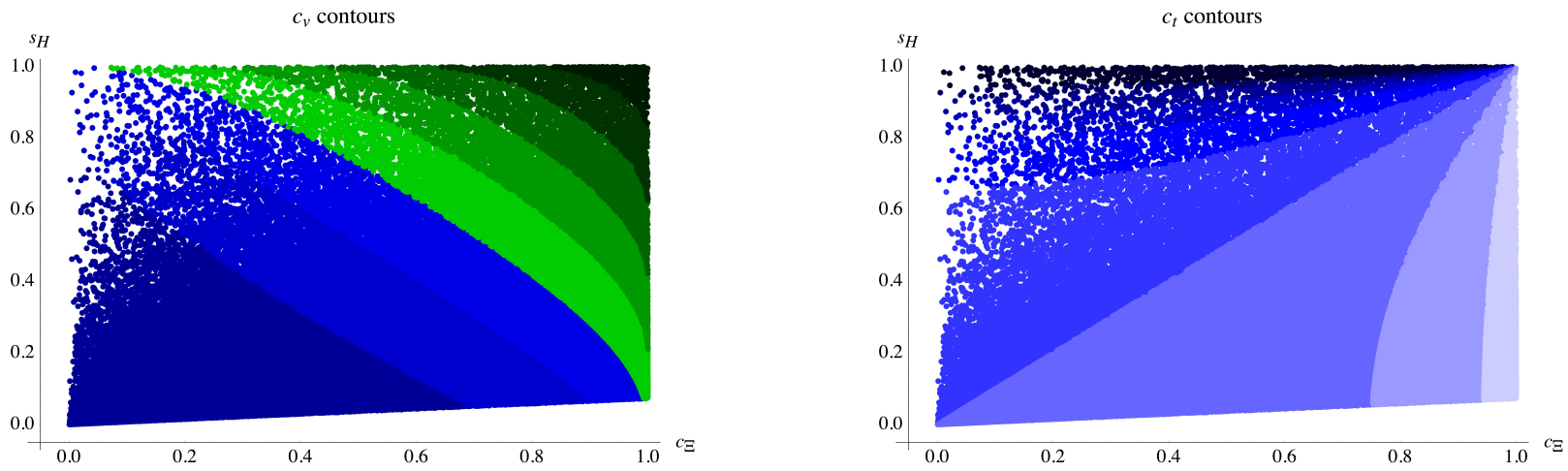

FIG. 2: Scan over the GM model's parameter space only requiring $m_{H_{0}} \simeq 126 \mathrm{GeV}$ in the $\left(c_{\Xi}, s_{H}\right)$-plane. The left panel shows $c_{v, H_{0}}$ contours with the following color codes: dark blue $-1<c_{v}<-0.66$, dark green $1.33<c_{v}<\sqrt{8 / 3}$. The right panel shows $c_{t, H_{0}}$ contours with dark blue $c_{t}<-3$, light blue $-0.33<c_{t}<0$.
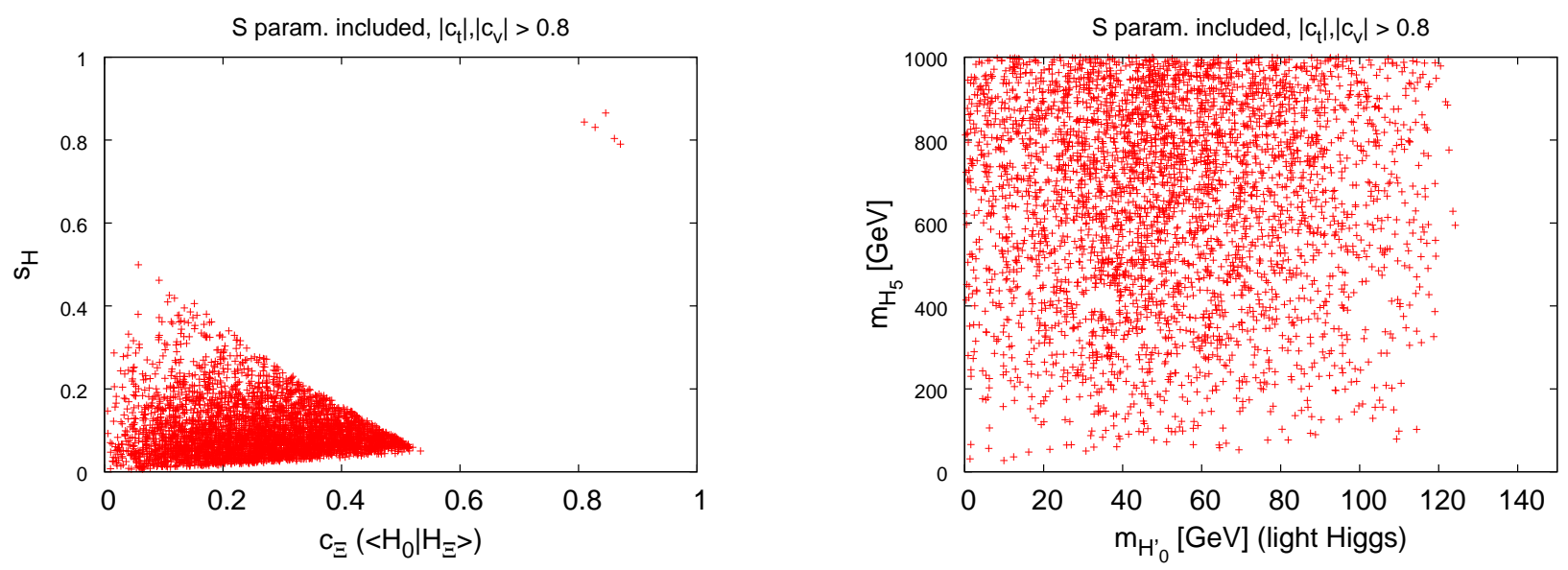

FIG. 3: Scan of Fig. 2, including precision constraints on the $S$ parameter.
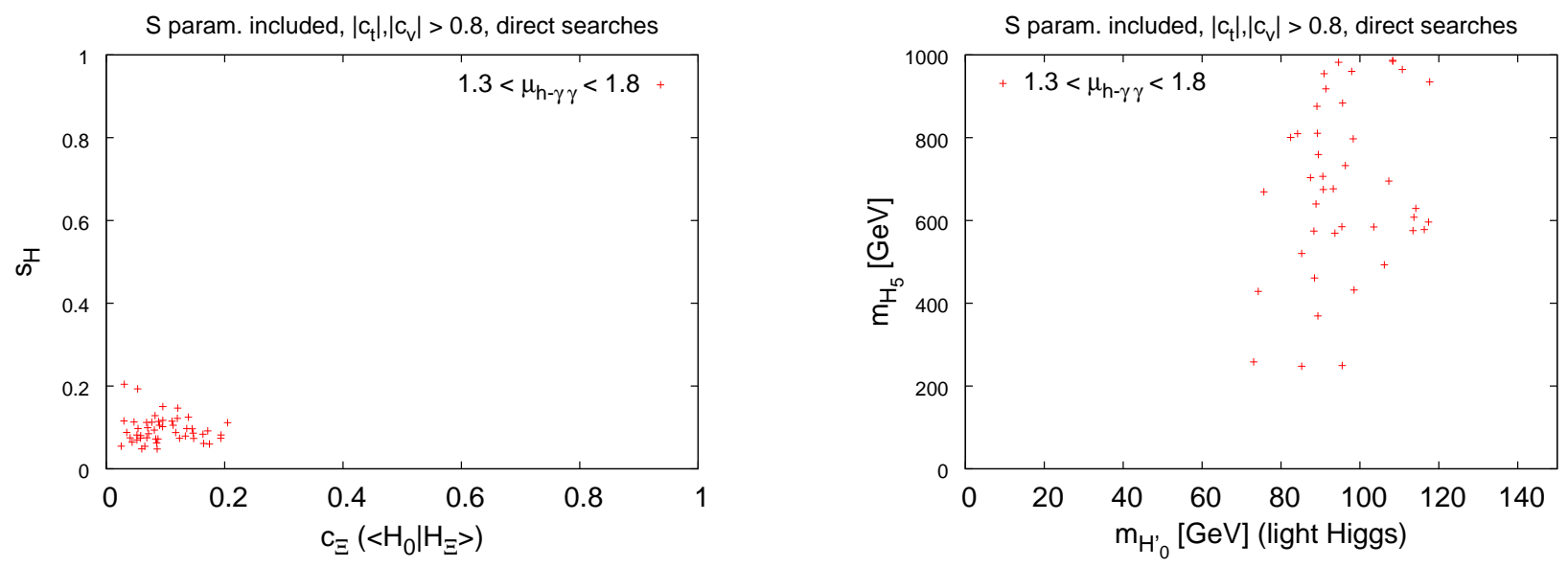

FIG. 4: Scan of Fig. 2, including precision constraints on the $S$ parameter and signal strength constraints from direct searches. 


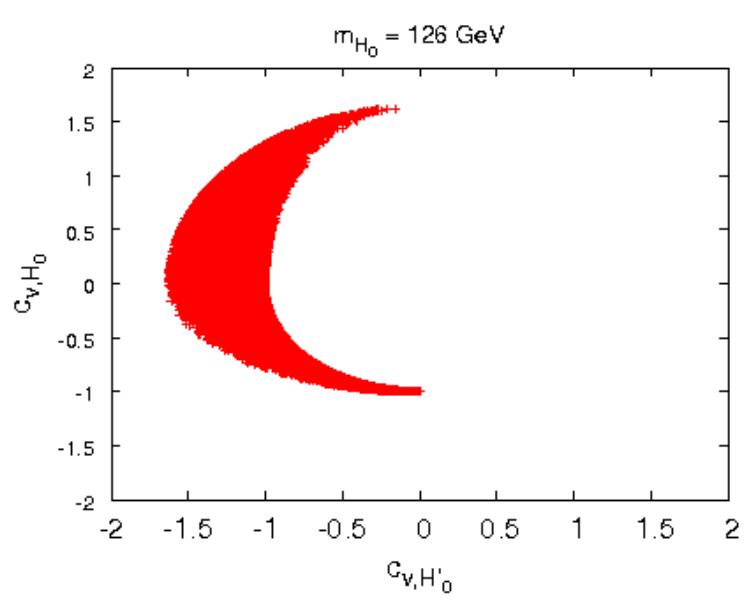

FIG. 5: $\quad\left(c_{v, H_{0}^{\prime}}, c_{v, H_{0}}\right)$ for $m_{H_{0}} \simeq 126 \mathrm{GeV}$. We impose neither electroweak precision constraints nor bounds from the signal strengths.

For later convenience, we also introduce the quantity

$$
c_{\Xi}=\left\{\begin{array}{l}
\left\langle H_{0}^{\prime} \mid H_{\Xi}\right\rangle=c_{q}, \text { if } m_{H_{0}^{\prime}} \simeq 126 \mathrm{GeV} \\
\left\langle H_{0} \mid H_{\Xi}\right\rangle=s_{q}, \text { if } m_{H_{0}} \simeq 126 \mathrm{GeV}
\end{array}\right.
$$

to quantify the overlap between the $126 \mathrm{GeV}$ mass eigenstate and $H_{\Xi}$, the singlet whose mass would only be generated by $v_{\Xi}$ if there was no mixing.

At this point we also note that with the conventions used in this work $c_{t}$ is always negative, whereas $c_{v}$ does not have a constrained sign. Although being different to the conventions often used by other groups, this is a perfectly legitimate choice that covers the physically relevant cases. As a consequence, the SM-like situation where the $h V V$ and $h f \bar{f}$ couplings have the same sign is recovered in this work when $\left(c_{t}, c_{v}\right)=(-1,-1)$.

\section{A. $\mathrm{m}_{\mathrm{H}_{0}} \simeq 126 \mathrm{GeV}$ - inverted mass hierarchy}

We first investigate the scenario where the Higgs candidate is more closely related to the SM Higgs doublet, i.e. $m_{H_{0}} \simeq 126 \mathrm{GeV}$. This will also provide us the munition for the phenomenologically more appealing case $m_{H_{0}^{\prime}} \simeq 126 \mathrm{GeV}$, for which there are no constraints from Higgs decays to light states with coupling strengths of the order of the weak scale.

The scenario with $m_{H_{0}} \simeq 126 \mathrm{GeV}$ corresponds to a situation which should be naively similar to the SM, because we expect that on average $m_{H_{0}} \simeq 126 \mathrm{GeV}$ can be easily obtained when the triplet vev is small, i.e. when Electroweak symmetry is mainly broken by the doublet. However, as we mentioned above, in this scenario we have $m_{H_{0}^{\prime}}$ smaller than $m_{H_{0}}$, which obviously implies peculiar consequences on the allowed phenomenology.

Fig. 1 shows the model's couplings spans in the $m_{H_{0}} \simeq$ $126 \mathrm{GeV}$ scenario, and in particular the allowed enlarged range for $c_{t}$ and $c_{v}$ described by Eq. 12. We start our walk through the constraints for $m_{H_{0}} \simeq 126 \mathrm{GeV}$ in Fig. 2, where no constraints have been imposed and the isocontours for $\left|c_{v, H_{0}}\right|$ and $\left|c_{t, H_{0}}\right|$ are shown in the $\left(c_{\Xi}, s_{H}\right)$ plane. In Fig. 3 we impose the $S$ parameter constraint and in the panels of Fig. 4 we further include the signal strengths of ATLAS. These steps sketch the transition from LEP to the combined Higgs discovery and exclusion.

As expected, due to $m_{H_{0}^{\prime}} \leq m_{H_{0}}$, we find large deviations from the SM Higgs decay phenomenology, which is reproduced within $\simeq 2 \sigma$ by the current data. In particular, we find that this feature holds even when we relax the constraints on the $T$ parameter: for negative $c_{v}$ values, $\left|c_{v}\right|$ is always smaller than 1 , in particular when $\left|c_{t}\right|$ is different from 1. This can be understood from the comments after Eq. (12): indeed, in this scenario, $s_{H}$ is usually relatively small, whereas $c_{\Xi} \sim 0$, i.e. $\left|c_{q}\right| \sim 1$. This means that there is room for $c_{t}$ to vary around the central value (remaining however close to -1 ), whereas the vector coupling essentially follows from $c_{q} c_{H}$. Therefore, $\left|c_{v}\right|$ is bound to be smaller than 1 . In particular, the more $c_{t}$ deviates to -1 , the smaller $\left|c_{v}\right|$ becomes. In such a situation, it is almost impossible to reproduce the Higgs signal: we typically find values $\mu_{h} \simeq 0.8$, whereas the value preferred by the excess observed by ATLAS is $\simeq 1.25$. The few surviving points shown in Fig. 4 correspond to $\mu_{h} \simeq 1$, which is just within the $1 \sigma$ error bar of the ATLAS global $\mu$ value. We also notice from the right plot in Fig. 4 that for all these points the decay $H_{0} \rightarrow H_{0}^{\prime} H_{0}^{\prime}$ is closed. On the other hand this means that a possible future decrease in the observed $h \rightarrow \gamma \gamma$ rate can in principle be accommodated by the GM triplet model in this scenario, at the price of some tension with electroweak precision measurements.

Since the only constraint we have required is the $S$ parameter along the lines of the previous section, it is natural to think that by relaxing the $S$ parameter condition to $99 \%$ confidence level, we can find points in the parameter space with values of $c_{t}$ and $c_{v}$ that allow to reproduce the Higgs signal. Indeed, we observe that there are points with larger values of $c_{t}$ and $c_{v}$ that survive the relaxed electroweak precision constraints. They typically imply larger values of $s_{H}$ and $c_{\Xi}$ values not necessarily close to 0 . Direct search constraints, however, both from LHC and LEP, remove these points, typically because they violate the LEP bound on the $H Z Z$ coupling for the light Higgs state, as can be readily seen from the left plot of Fig. 1. values of $c_{v, H_{0}} \geq 1$ correspond to values of $\left|c_{v, H_{0}^{\prime}}\right|$ that are too large to survive LEP bounds.

This scenario, seems to be heavily constrained by the $S, T, U$ parameters on top of the phenomenological requirements for the observed signal strengths. The latter is expected from our previous remarks on dominant decays to the lighter Higgs states, however, one might naively expect that electroweak precision should not be too constraining as this is definitely not the case for the model's limit of the SM Higgs doublet with $m_{h} \simeq$ 
$126 \mathrm{GeV}$ 45. So what is the reason for electroweak precision observables being so different from the SM in this case?

Let us step back and investigate how electroweak precision observables are qualitatively influenced in the GM model. In comparison to the SM the electroweak precision observables are influenced by the modified Higgs sector. The gauge interactions of the fermions are unchanged and by comparing to the SM reference point to calculate $\Delta S, \Delta T, \Delta U$ drop out. To understand the Higgs-gauge boson interactions which drive these observables via Eq. (14), it is quite instrumental to understand how unitarity conservation is realized in longitudinal gauge boson scattering in the GM model. Looking at, e.g., $W_{L} W_{L}$ scattering, a necessary condition for unitarity to be conserved in any perturbative model is that the coherent sum of new physics contributions to $W W \rightarrow W W$ has to reproduce the SM Higgs contribution for high enough energies (above all contributing thresholds). The $s$ and $t$-channel SM Higgs exchange cancels the residual amplitudes growth proportional to the the $W$ 's energy squared in a minimal fashion. In the GM model this is realized more intricately as, e.g., in models with just simple Higgs mixing. While in the latter case, for high enough energies, the SM Higgs contribution is reproduced via $\sin ^{2} \alpha+\cos ^{2} \alpha=1$ ( $\alpha$ is the mixing angle), in the GM model we can have a very large enhancement of $c_{v}$ by all uncharged Higgs particles to begin with. Their "over-contribution" is cancelled by $t$ - and $u$-channel exchange of the $H_{5}^{ \pm \pm}$which in the high energy limit becomes equivalent to an $s$-channel contribution [16. Note that all Higgs exchange diagrams are proportional to the squared real Higgs couplings and the compensation results from kinematics $t, u \sim-s$. The two-point functions of Eq. (14) obviously do not encode any kinematics and are a coherent sum of the quartic Higgs couplings and the trilinear couplings squared. Spontaneously broken gauge invariance, on the other hand, guarantees the absence of UV singularities via cancellations among the different contributing diagrams (and the corresponding $T$ parameter counter term).

From Fig. 5 we can therefore immediately read off the potential issue of the GM model that arises when it is confronted with electroweak precision measurements for $m_{H_{0}} \simeq 126 \mathrm{GeV}$. There we plot the $\left(c_{v, H_{0}^{\prime}}, c_{v, H_{0}}\right)$ correlation for our scan with the requirement of $H_{0}$ being the observed Higgs-like candidate. Obviously there is no anti-correlation of the two Higgs states. When enforcing the observed data's constraint on $\left|c_{v, H_{0}}\right| \simeq 1$ (horizontal lines in Fig. 5) we typically have sizeable values for $c_{v, H_{0}^{\prime}}$ : therefore $\Delta S$ generically turns out to be large when we require the Higgs candidate's couplings to reproduce the SM or to obtain even larger couplings than the SM, $c_{v, H_{0}} \gtrsim 1$. This together with the large deviation of the Higgs phenomenology driven by $\operatorname{BR}\left(H_{0} \rightarrow H_{0}^{\prime} H_{0}^{\prime}\right)$ highly constrains $m_{H_{0}} \simeq 126 \mathrm{GeV}$, independent of a possible excess in $\operatorname{BR}\left(H_{0} \rightarrow \gamma \gamma\right)$.

\section{B. $\mathbf{m}_{\mathrm{H}_{0}^{\prime}} \simeq 126 \mathrm{GeV}$ - normal mass hierarchy}

We turn to identifying $H_{0}^{\prime}$ with the observed Higgs candidate. In Fig. 7 we plot the different contour regions for $c_{v, H_{0}}$ and $c_{t, H_{0}}$ as functions of $\left(c_{v, H_{0}^{\prime}}, c_{t, H_{0}^{\prime}}\right)$ without imposing any constraints apart from $m_{H_{0}^{\prime}} \simeq 126 \mathrm{GeV}$. The left-out region inside the blue contours stems from parameter points that do not give rise to (local) minimum of the potential. The model clearly predicts large enhancements of the vectorial couplings, which also manifest in an enhanced partial decay width $\Gamma\left(H_{0}^{\prime} \rightarrow \gamma \gamma\right)$, which is a function of basic contributions: the $W$ loop, the fermion loops and the charged scalar loops. In the SM the $W$ loop dominates over the top contributions. Fig. 7 indicates that this correlation is already significantly altered via Eq. (12): the top loop can be suppressed while the $W$ loop is enhanced. This needs to be contrasted to ordinary complex triplet models, where, due to the small triplet vev, such an enhancement via $c_{v}$ is not present and possible branching ratio enhancements need to be driven essentially only by the additional charged scalars.

The contribution from e.g. charged $H_{5}^{ \pm}$and $H_{5}^{ \pm \pm}$ scalars in the GM model to the $H_{0}^{\prime} \rightarrow \gamma \gamma$ amplitude scales as $F\left(s_{H}, \vec{\lambda}\right) Q_{h_{5}}^{2}\left[v_{\mathrm{SM}}^{2} /\left(2 m_{5}^{2}\right)\right] A_{0}\left(m_{H_{0}^{\prime}}^{2} / 4 m_{H_{5}}^{2}\right)$, where $F$ is a function of the parameter space and $A_{0}$ is the typical one-loop 3-point function encountered in such amplitudes [35]. A similar relation holds for $H_{3}^{ \pm}$. The 3-point function is essentially constant for masses larger than $m_{H_{5}} \gtrsim 200 \mathrm{GeV}, A_{0} \simeq 0.35$. In the end the charged scalar effect can be compensated by the vast parameter range that is admissible due to changes in $\left(c_{v}, c_{t}\right)$, which can also constructively interfere as a consequence of Eq. 12 . We find that the $H_{5}$ contribution is typically small and destructive over the parameter range that we consider, i.e. it works against the $W$ contribution; this means that a necessary condition to have

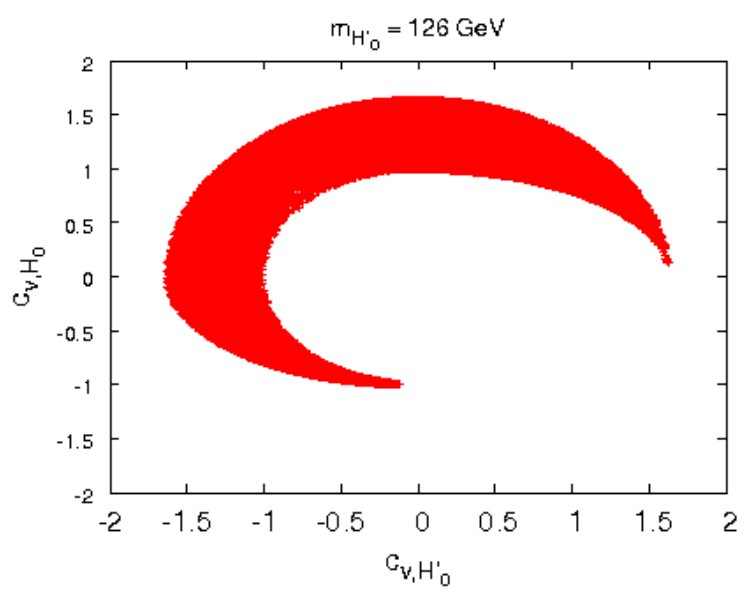

FIG. 6: $\quad\left(c_{v, H_{0}^{\prime}}, c_{v, H_{0}}\right)$ for $m_{H_{0}^{\prime}} \simeq 126 \mathrm{GeV}$. We impose neither electroweak precision constraints nor bounds from the signal strengths. 

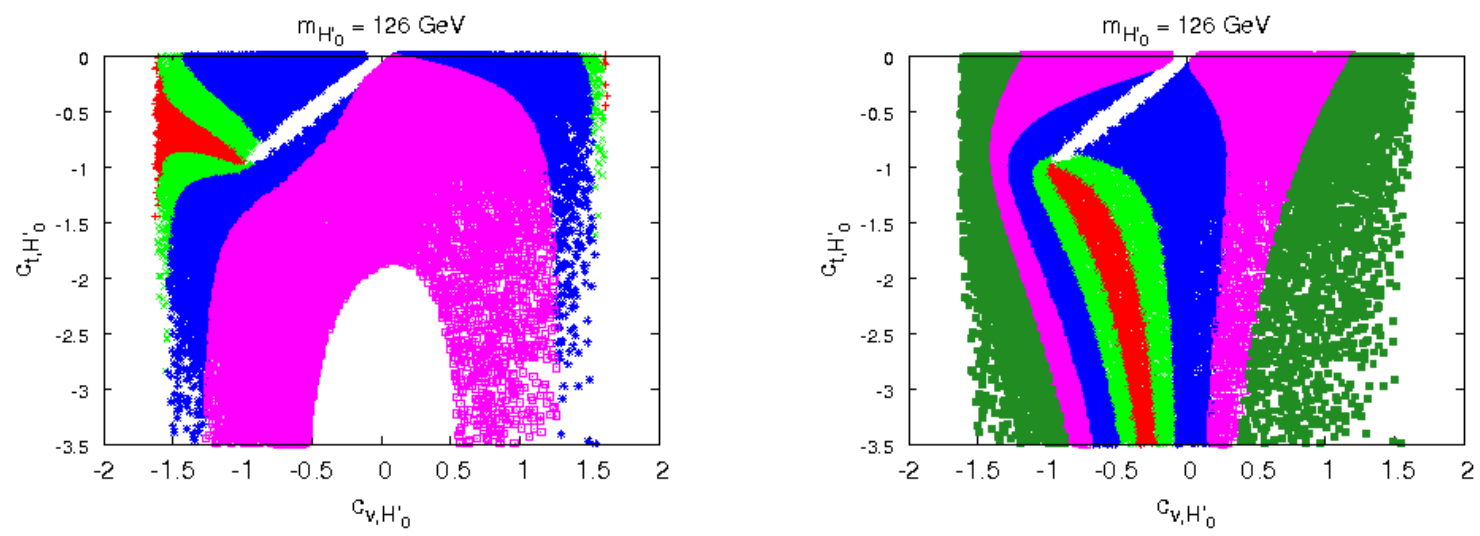

FIG. 7: $\quad\left(c_{v, H_{0}^{\prime}}, c_{t, H_{0}^{\prime}}\right)$ correlation for $m_{H_{0}^{\prime}} \simeq 126 \mathrm{GeV}$. We do not impose any other additional constraints. The color code is the same as in Fig. 1] although here contours are for $c_{v, H_{0}}$ (on the left panel) and $c_{t, H_{0}}$.

$\operatorname{BR}\left(H_{0}^{\prime} \rightarrow \gamma \gamma\right) \gtrsim \mathrm{BR}\left(H_{0}^{\prime} \rightarrow \gamma \gamma\right)_{\mathrm{SM}}$ is that the destructive interference of the SM $t$ and $W^{ \pm}$is reduced or becomes constructive.

We again start our walk through the constraints for $m_{H_{0}^{\prime}} \simeq 126 \mathrm{GeV}$ in Fig. 8. In Fig. 9 we impose the $S$ parameter constraint and in the panels of Fig. 10 we include the signal strengths of ATLAS. We show the fully tuned setup in the analogous Figs. 11 and 12 . From Fig. 6 we expect the $m_{H_{0}^{\prime}} \simeq 126 \mathrm{GeV}$ scenario to be less sensitive to electroweak precision constraints: $\left|c_{v, H_{0}^{\prime}}\right| \sim 1$ (vertical lines in Fig. 6) allows for a wide range on $c_{v, H_{0}}$.

Indeed, by enforcing electroweak precision constraints we find our results to be also less sensitive to the $T$ parameter, although more parameter points at large $s_{H}$ are rejected. Nonetheless we still obtain consistent parameter choice for large values of $s_{H}$, which as consequence of signal strengths then needs to be anti-correlated to $c_{\Xi}$. The projection on $\left(c_{\Xi}, s_{H}\right)$ therefore nicely discriminates between different regions of diphoton branching ratio enhancements. We again see that a potential excess in the diphoton final state in the GM model is predominantly realized via relaxing the SM-constraints on couplings to the top and massive gauge boson sector with the additional scalars providing some additional freedom in this respect, yet their impact is subdominant unless $m_{H_{5}}$ is significantly small.

A generic prediction of the GM model when it reproduces the current data and does not violate electroweak constraints on $S, T$ is the presence of a relatively heavy quintet. The triplet state are also heavy but their phenomenology is sufficiently suppressed (keep in mind that we impose ATLAS exclusion contours on the uncharged states throughout). The quintet's production is suppressed but given the mass of this state we expect a measurement of the phenomenologically clean same-sign $W$ production via $\mathrm{WBF}$ to give substantial constraints on the realization $m_{H_{0}^{\prime}} \simeq 126 \mathrm{GeV}$. Adapted searches will therefore have the potential to further constrain the GM model's allowed parameter space, or, conversely, to find an hint of a doubly charged scalar.

$$
\text { Heavy triplet — light quintet }
$$

Until now our parameter choices are dominated by choices such that the quintet states typically outweigh the triplet Higgs bosons. Now, we specifically analyze the situation when the triplet is heavy $\left(m_{H, 3} \simeq 500 \mathrm{GeV}\right)$ and the quintet states are light $m_{H_{5}}<m_{H_{3}}$. The resulting spectra with a heavy triplet and a relatively light quintet should be favored by flavor analyses, since the quintet is fermiophobic and the triplet is less related to fermion mass generation and should hence decouple for large masses from flavor observables, when $s_{H}$ is not too large. We will also get a better understanding of the correlation between $s_{H}$ and $c_{q}$.

As shown in Figs. 13 and 14 this dedicated scan projects out the values of $s_{H} \simeq 1$ with typically heavy additional singlets $H_{0}$ in the range of several $100 \mathrm{GeV}$. With respect to our previous remarks in Sec. IVB about how $H_{0}^{\prime} \rightarrow \gamma \gamma$ comes about, we do not find any notable qualitative modification of our earlier findings: the enhanced diphoton branching is still predominantly enabled via the interplay of $c_{v, H_{0}^{\prime}}$ and $c_{t, H_{0}^{\prime}}$ and potentially large branching ratios can be achieved. These parameter choices typically imply a very large $H_{0}$ mass compared to the other case - future searches for uncharged SM Higgs-like states in the hundreds of $\mathrm{GeV}$ range will highly constrain this fact of the GM model.

\section{SM-like GM phenomenology}

Let us abandon the diphoton excess initially observed by both ATLAS and CMS and briefly investigate the parameter range for which $\mathrm{BR}\left(H_{0}^{\prime} \rightarrow \gamma \gamma\right) \simeq \mathrm{BR}(h \rightarrow$ $\gamma \gamma)_{\mathrm{SM}}$. This amounts to a potential future measurement which shows an even larger resemblance to the SM 

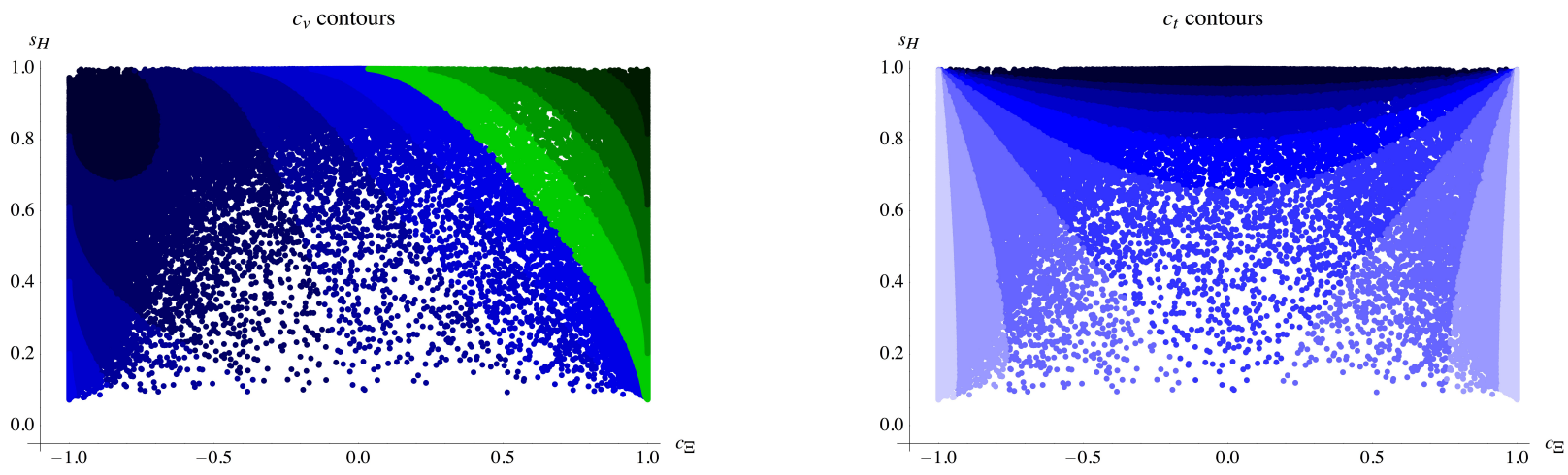

FIG. 8: Scan over the GM models parameter space only requiring $m_{H_{0}^{\prime}} \simeq 126 \mathrm{GeV}$ in the $\left(c_{\Xi}, s_{H}\right)$-plane. The left panel shows $c_{v, H_{0}^{\prime}}$ contours with the following color codes: dark blue $-\sqrt{8 / 3}<c_{v}<-1.33$, dark green $1.33<c_{v}<\sqrt{8 / 3}$. The right panel gives $c_{t, H_{0}^{\prime}}$ contours with dark blue $c_{t}<-3$, light blue $-0.33<c_{t}<0$.
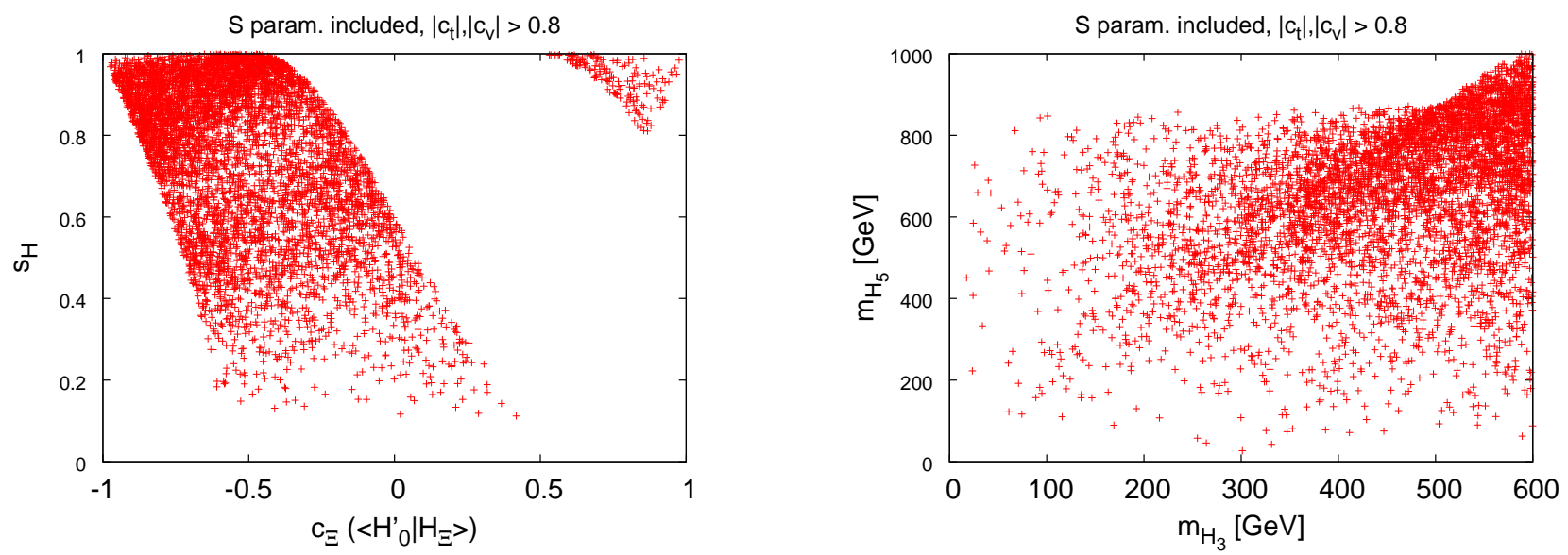

FIG. 9: Scan of Fig. 8, including precision constraints on the $S$ parameter.
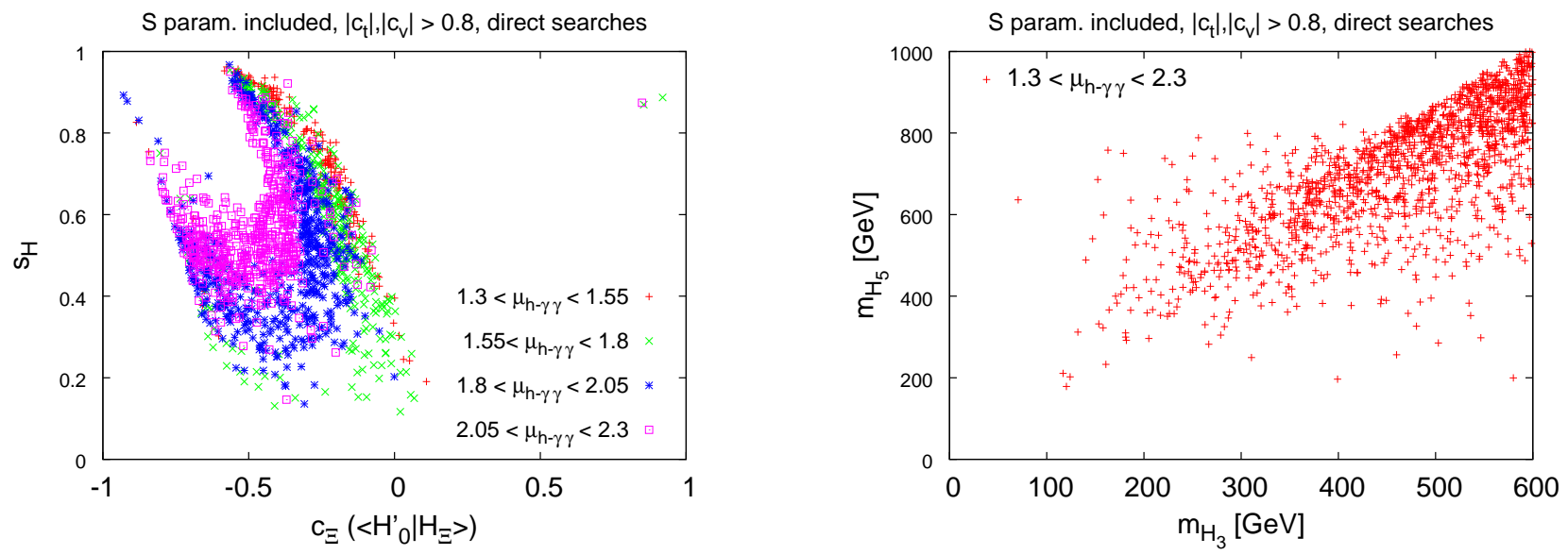

FIG. 10: Scan of Fig. 8 including precision constraints on the $S$ parameter and signal strength constraints from direct searches. 

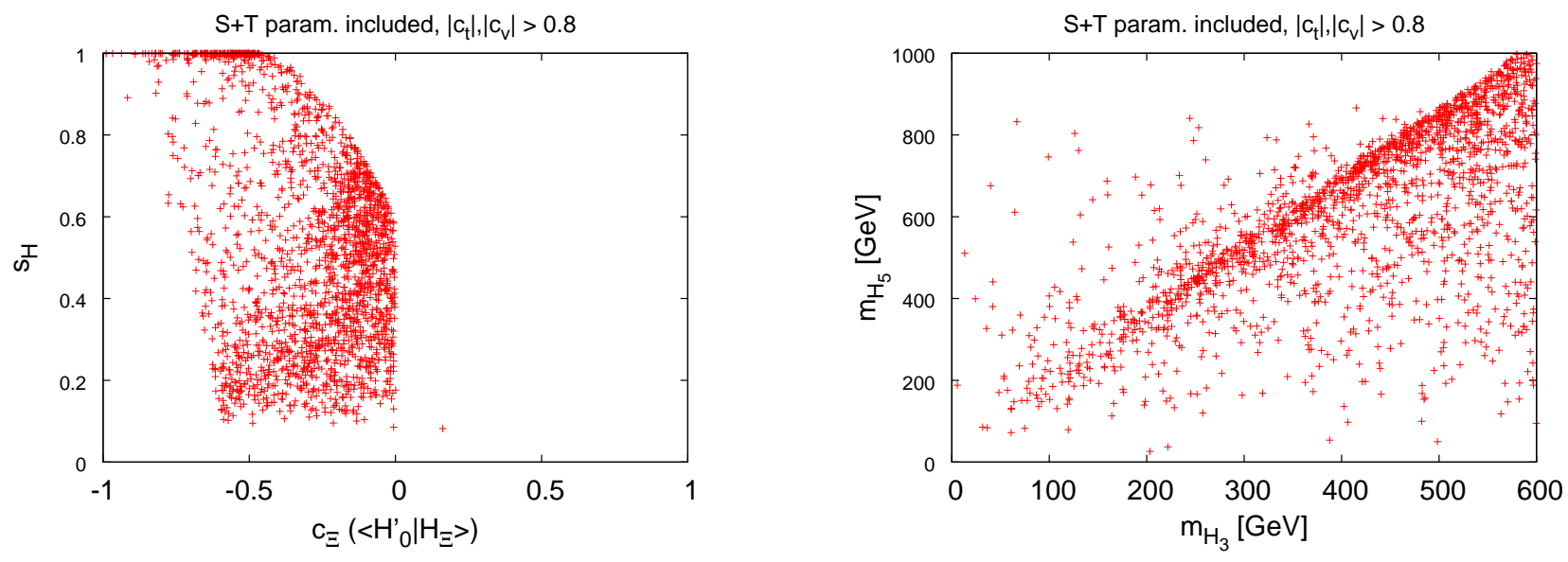

FIG. 11: Scan of Fig. 8 including precision constraints on both the $S$ and the $T$ parameters.
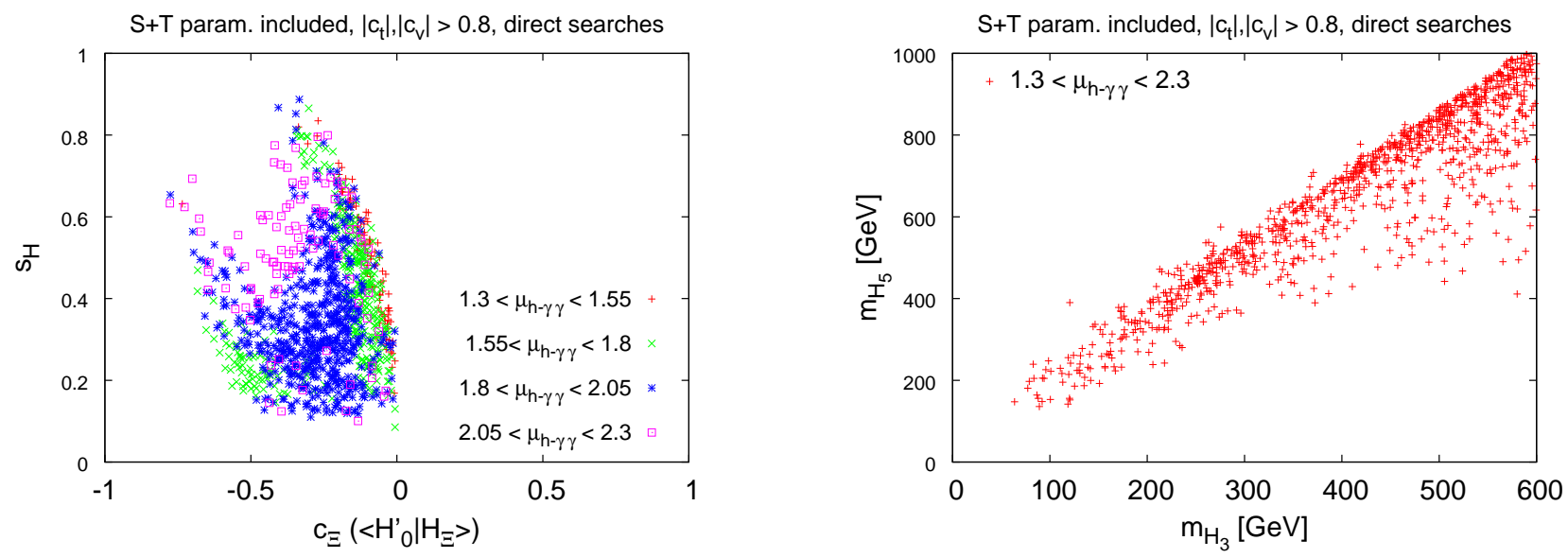

FIG. 12: Results of Fig. 10 including constraints on the $S$ and $T$ parameters, and signal strength constraints from direct searches.
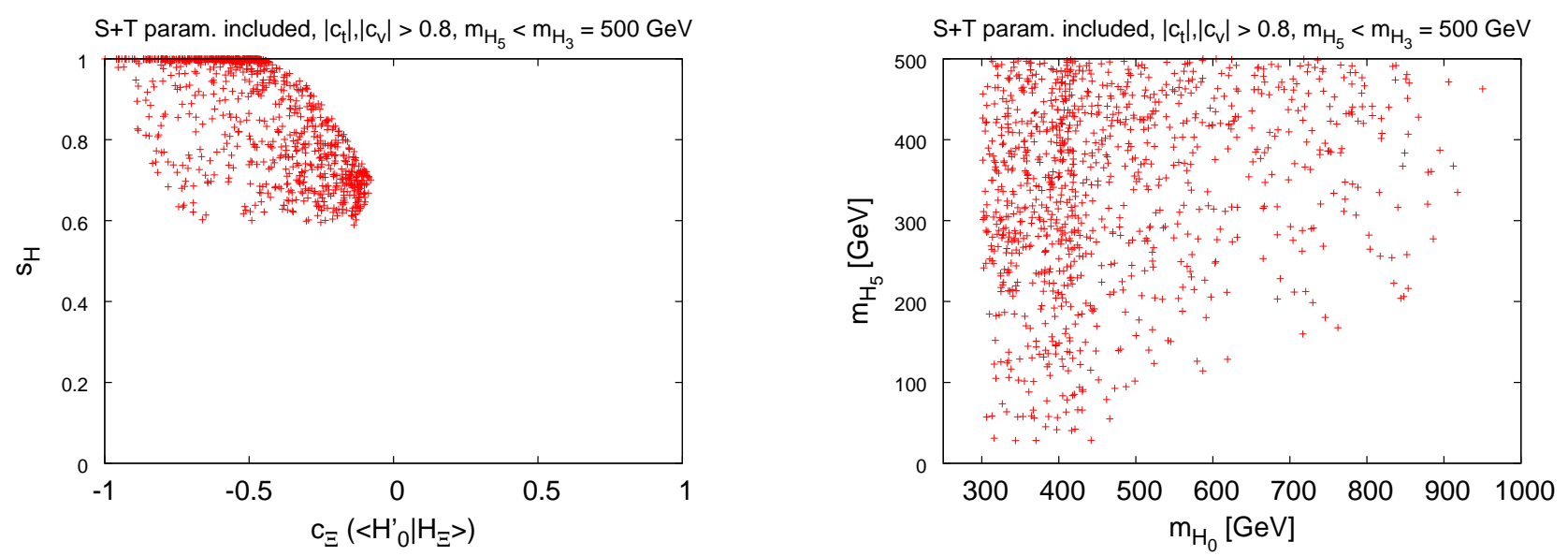

FIG. 13: Dedicated scan with $m_{H_{3}}=500 \mathrm{GeV}$ and $m_{H_{5}}<m_{H_{3}}$ while $m_{H_{0}^{\prime}} \simeq 126 \mathrm{GeV}$. Constraints on both the $S$ and $T$ parameters are included. 

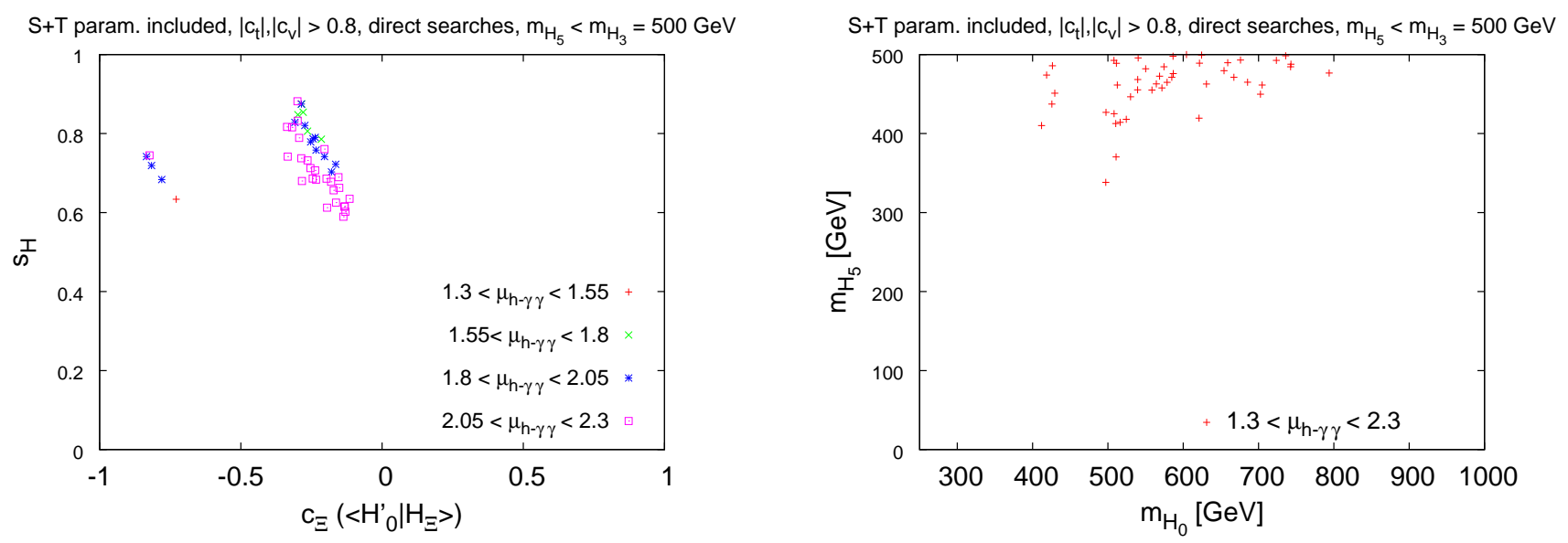

FIG. 14: Signal strengths included on top of the results of Fig. 13 .

than we currently observe. Parameter choices of the GM model with such a measurement would be required to be more tailored to the SM. Identifying $H_{0}$ as our Higgs candidate is obviously still disfavored along the lines of our discussion of Sec. IV A. Furthermore, from our analysis of the $m_{H_{0}^{\prime}} \simeq 126 \mathrm{GeV}$ it should be clear that the number of degrees of freedom in the light of not too constraining Higgs searches for masses different than $126 \mathrm{GeV}$ and the available parameter space are large enough to account for such a situation (a limit of $\sigma \times \mathrm{Br} /[\sigma \times \mathrm{Br}]_{\mathrm{SM}} \simeq 0.1$ roughly corresponds to $\left|c_{v}\right| \lesssim 0.32$ ). This is especially true for parameter choices for which an additional scalar falls into the vicinity of the observed $126 \mathrm{GeV}$ Higgs candidate, where resolution effects limit stringent constraints. We assume consistency with the SM hypothesis within $20 \%$ of the $W W, Z Z, \gamma \gamma$ and combined categories in the following, but we keep the ATLAS constraints on the other Higgs states unmodified. With our remarks about the $\left(c_{\Xi}, s_{H}\right)$ plane being a good discriminant of the diphoton enhancement in mind we observe in Fig. 15 and 16 that the SM-like requirements slice out a specific parameter region in the GM model (independent of the $T$ parameter).

This teaches two important lessons for an observation of perfectly consistent SM Higgs measurements in the future. On the one hand, even if such an outcome does not speak in favor of the GM model, there is still a lot of parameter space available, which can and needs to be tested at the LHC. From this point of view, it again appears indispensable to extend existing Higgs-like searches to the heavy mass regime while relaxing specific assumptions on the total particle width as limiting factor of such analyses. With again quintet masses in the hundreds of $\mathrm{GeV}$ regime, which can be straightforwardly accessed at the LHC in same-sign $W$ final states, the GM model can be highly constrained, even when its Higgs candidate phenomenology highly resembles the SM Higgs.

\section{CONCLUSIONS}

The Georgi-Machacek model implements Higgs triplets in a custodially invariant way at the price of an additional fine tuning problem. In this paper we have performed an analysis of the model's parameter space in the light of direct collider and electroweak precision constraints. Depending on the interpretation of electroweak precision in the GM model context, there are important consequences for the model when it is confronted with data. Given that the GM model has a wide range of coupling spans, excesses in the $h \rightarrow \gamma \gamma$ rate can be observed, but there is also a vast parameter region that is allowed if data resembles the SM.

In addition to the SM, the GM model introduces a number of new scalars. However, in the light of recent measurements only a subset of these can be considered the discovered Higgs candidate. One peculiarity that arises in the GM model is that if the Higgs boson that arises mostly from the SM-like doublet Higgs field is identified with the Higgs candidate at around $126 \mathrm{GeV}$, there is a light scalar in the spectrum which mostly arises from the triplet. This puts strong constraints on this option as branching ratios to SM matter quickly deviate from their observed values when the light scalar becomes accessible as a Higgs decay channel. Furthermore, electroweak precision constraints (mostly by the $S$ parameter) disfavor this option even if the light scalar is not constrained by LEP measurements. If the excess in the diphoton channel prevails this option becomes heavily constrained whereas lower combined signal strengths can be obtained at the cost of a tension with electroweak precision data.

Most of these constraints are relaxed when we identify the non-doublet state with the observed candidate. Here a vast parameter space becomes available to accommodate excesses in the $\gamma \gamma$ channel, but the model also exhibits parameter regions consistent with SM.

For our simplified lagrangian approach, the consistent 

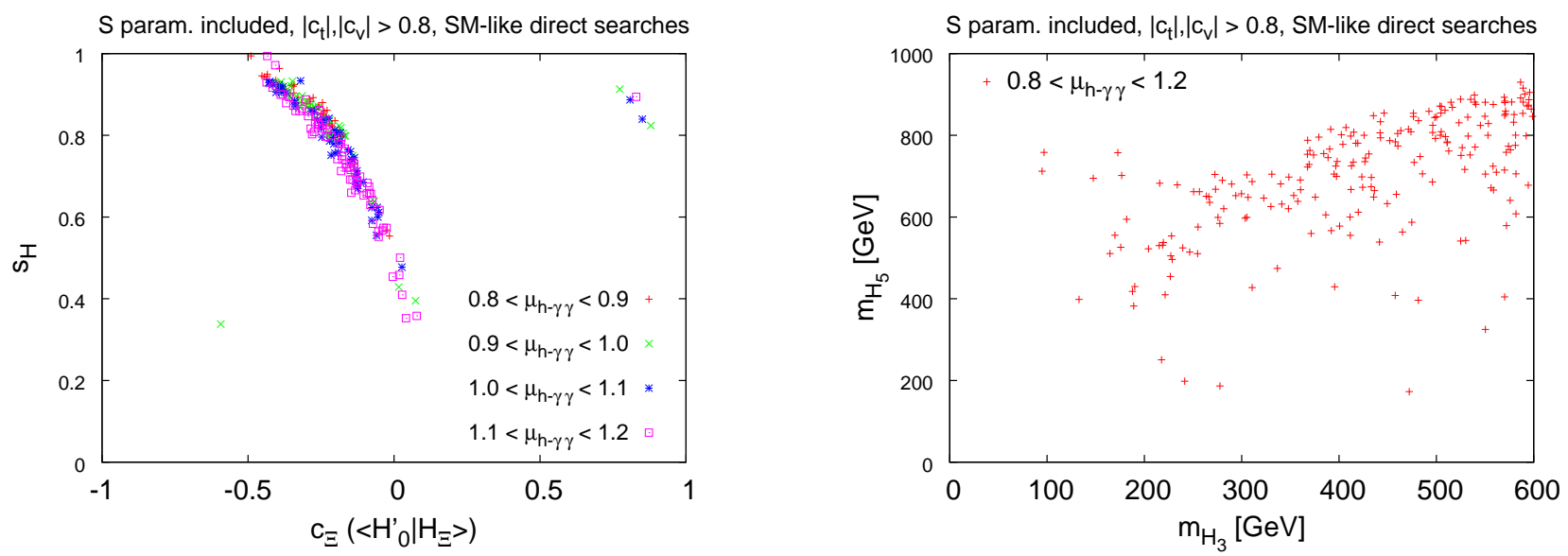

FIG. 15: Scan of Fig. 8, including precision constraints on the $S$ parameter, and signal strength constraints, in a SM-like scenario where $\mu_{h \rightarrow W W}, \mu_{h \rightarrow \gamma \gamma}$ and the total $\mu$ value lie in the interval $[0.8,1.2]$.
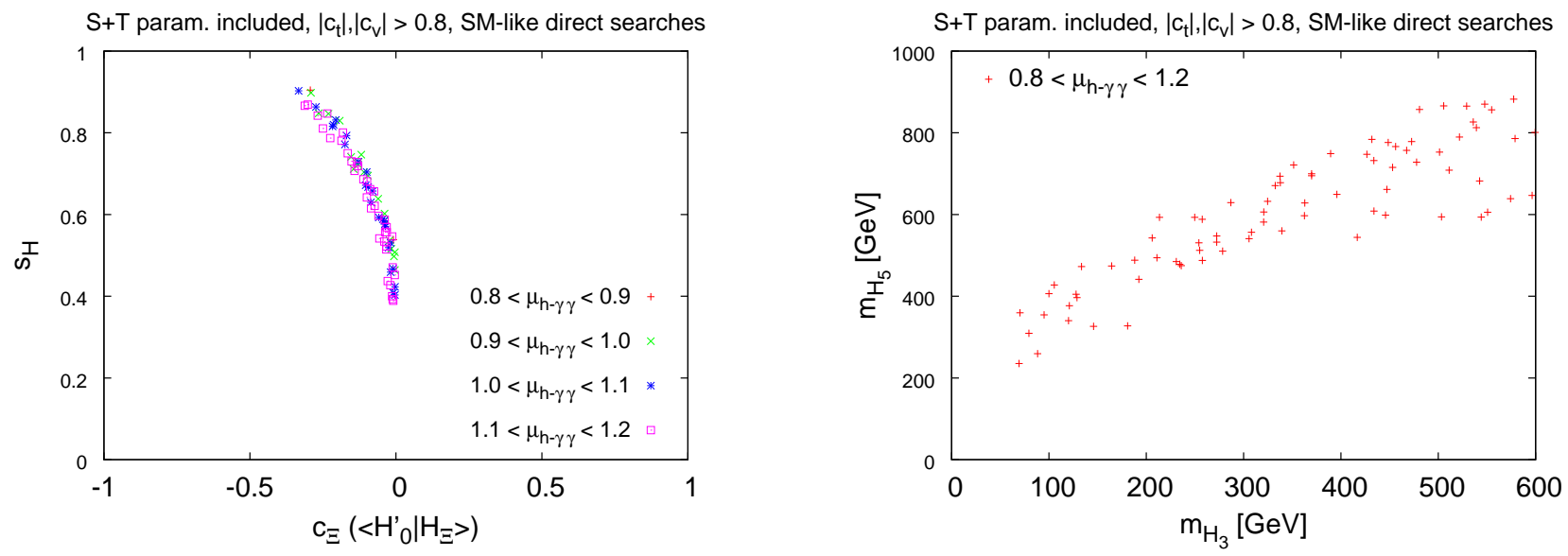

FIG. 16: Scan of Fig. 8, including precision constraints on the $S$ and $T$ parameters, and signal strength constraints, in a SM-like scenario where $\mu_{h \rightarrow W W}, \mu_{h \rightarrow \gamma \gamma}$ and the total $\mu$ value lie in the interval $[0.8,1.2]$.

parameter choices of the SM typically predict the presence of new states in the hundreds of $\mathrm{GeV}$ region. Lighter spectra are admissible by current collider constraints but typically result from extremely small mixing of the custodial triplets, one of which giving rise to massive gauge bosons. While the triplet mass eigenstate is $\mathcal{C P}$ odd and can only be produced via gluon fusion, the quintet state is fermiophobic and can be constrained via a dedicated measurement in WBF-type production in the near future. The latter search is likely to put extremely tight constraints on the GM model, especially if future mea- surements of the $126 \mathrm{GeV}$ Higgs candidate are consistent with the SM predictions.

\section{Acknowledgments}

We would like to thank Claude Duhr for FEYNRules support. CE thanks Adam Falkowski and Jure Zupan for discussions during the CERN BSM Theory Summer Institute. ER thanks Uli Haisch for useful discussions.

CE acknowledges funding by the Durham International Junior Research Fellowship scheme.
[1] F. Englert and R. Brout, Phys. Rev. Lett. 13 (1964) 321. P. W. Higgs, Phys. Lett. 12 (1964) 132 and Phys. Rev. Lett. 13 (1964) 508. G. S. Guralnik, C. R. Hagen and T. W. B. Kibble, Phys. Rev. Lett. 13 (1964) 585.
[2] The ATLAS collaboration, Phys. Lett. B 716 (2012) 1.

[3] The CMS collaboration, Phys. Lett. B 716 (2012) 30.

[4] The ATLAS collaboration, ATLAS-CONF-2012-170.

[5] The CMS collaboration, CMS-PAS-HIG-12-045. 
[6] S. Chang, C. A. Newby, N. Raj and C. Wanotayaroj, Phys. Rev. D 86 (2012) 095015.

[7] A. G. Akeroyd and S. Moretti, Phys. Rev. D 86 (2012) 035015

[8] D. Carmi, A. Falkowski, E. Kuflik, T. Volansky and J. Zupan, JHEP 1210, 196 (2012).

[9] M. E. Peskin and T. Takeuchi, Phys. Rev. Lett. 65 (1990) 964 and Phys. Rev. D 46 (1992) 381.

[10] H. Georgi and M. Machacek, Nucl. Phys. B 262 (1985) 463. M. S. Chanowitz and M. Golden, Phys. Lett. B 165 (1985) 105.

[11] K. Cheung and D. K. Ghosh, JHEP 0211 (2002) 048.

[12] S. Godfrey and K. Moats, Phys. Rev. D 81, 075026 (2010).

[13] M. Chaichian, P. Hoyer, K. Huitu, V. A. Khoze and A. D. Pilkington, JHEP 0905 (2009) 011.

[14] L. Wang and X. -F. Han, Phys. Rev. D 86, 095007 (2012) and Phys. Rev. D 87, 015015 (2013).

[15] H. E. Logan and M. -A. Roy, Phys. Rev. D 82, 115011 (2010).

[16] J. F. Gunion, R. Vega and J. Wudka, Phys. Rev. D 42, 1673 (1990).

[17] A. Djouadi, W. Kilian, M. Muhlleitner and P. M. Zerwas, Eur. Phys. J. C 10 (1999) 27. U. Baur, T. Plehn and D. L. Rainwater, Phys. Rev. D 69 (2004) 053004. M. J. Dolan, C. Englert and M. Spannowsky, JHEP 1210 (2012) 112. F. Goertz, A. Papaefstathiou, L. L. Yang and J. Zurita, arXiv:1301.3492 [hep-ph].

[18] G. 't Hooft, NATO Adv. Study Inst. Ser. B Phys. 59 (1980) 135.

[19] A. Arhrib, R. Benbrik, M. Chabab, G. Moultaka and L. Rahili, JHEP 1204, 136 (2012).

[20] N. D. Christensen and C. Duhr, Comput. Phys. Commun. 180 (2009) 1614.

[21] A. G. Akeroyd, Phys. Lett. B 353 (1995) 519.

[22] J. F. Gunion, P. Kalyniak, M. Soldate and P. Galison, Phys. Rev. Lett. 54 (1985) 1226.

[23] J. F. Gunion, R. Vega and J. Wudka, Phys. Rev. D 43, 2322 (1991).

[24] C. Wetterich, Phys. Lett. B 140 (1984) 215.

[25] R. Barbieri, A. Pomarol, R. Rattazzi and A. Strumia, Nucl. Phys. B 703 (2004) 127.

[26] V. D. Barger, J. L. Hewett and R. J. N. Phillips, Phys. Rev. D 41 (1990) 3421. J. F. Gunion and H. E. Haber, Phys. Rev. D 67 (2003) 075019.

[27] H. E. Haber and H. E. Logan, Phys. Rev. D 62 (2000) 015011.

[28] The CMS collaboration, arXiv:1207.2666 [hep-ex]. The ATLAS collaboration, arXiv:1210.5070 [hep-ex].

[29] The CMS collaboration, Phys. Rev. Lett. 109, 071803 (2012) and CMS-PAS-SUS-12-017. The ATLAS collaboration, JHEP 1110 (2011) 107.

[30] D. Goncalves-Netto, D. Lopez-Val, K. Mawatari,
T. Plehn and I. Wigmore, arXiv:1211.0286 [hep-ph]

[31] A. G. Akeroyd, M. Aoki and H. Sugiyama, Phys. Rev. D 79 (2009) 113010.

[32] M. Kakizaki, Y. Ogura and F. Shima, Phys. Lett. B 566 (2003) 210.

[33] W. Konetschny and W. Kummer, Phys. Lett. B 70, 433 (1977).

[34] The CMS collaboration, HIG-12-041.

[35] H. M. Georgi, S. L. Glashow, M. E. Machacek and D. V. Nanopoulos, Phys. Rev. Lett. 40 (1978) 692D. A. Djouadi, M. Spira and P. M. Zerwas, Phys. Lett. B 264 (1991) 440. S. Dawson, Nucl. Phys. B 359, 283 (1991). M. Spira, A. Djouadi, D. Graudenz and P. M. Zerwas, Nucl. Phys. B 453 (1995) 17. R. V. Harlander and W. B. Kilgore, Phys. Rev. Lett. 88, 201801 (2002). S. Moch and A. Vogt, Phys. Lett. B 631, 48 (2005).

[36] D. L. Rainwater and D. Zeppenfeld, JHEP 9712 (1997) 005. D. L. Rainwater, D. Zeppenfeld and K. Hagiwara, Phys. Rev. D 59 (1998) 014037. T. Figy, D. Zeppenfeld, and C. Oleari Phys. Rev. D 68 (2003) 073005. for electroweak corrections in the context of the SM electroweak sector see M. Ciccolini, A. Denner and S. Dittmaier, Phys. Rev. Lett. 99 (2007) 161803.

[37] S. Dittmaier et al. [LHC Higgs Cross Section Working Group Collaboration], arXiv:1101.0593 [hep-ph].

[38] The ATLAS collaboration, ATLAS-CONF-2012-127.

[39] R. Barate et al. [LEP Working Group for Higgs boson searches and ALEPH, DELPHI, L3 and OPAL Collabs], Phys. Lett. B 565 (2003) 61.

[40] The CMS collaboration, CMS-HIG-12-045. The ATLAS collaboration, ATLAS-CONF-2012-127.

[41] A. Azatov, R. Contino and J. Galloway, JHEP 1204 (2012) 127. P. P. Giardino, K. Kannike, M. Raidal and A. Strumia, Phys. Lett. B $\mathbf{7 1 8}$ (2012) 469. J. Ellis and T. You, JHEP 1209 (2012) 123. J. R. Espinosa, C. Grojean, M. Muhlleitner and M. Trott, JHEP 1212 (2012) 045. T. Plehn and M. Rauch, Europhys. Lett. 100, 11002 (2012). G. Moreau, Phys. Rev. D 87, 015027 (2013). T. Corbett, O. J. P. Eboli, J. Gonzalez-Fraile and M. C. Gonzalez-Garcia, arXiv:1211.4580 [hep-ph]. E. Masso and V. Sanz, arXiv:1211.1320 [hep-ph].

[42] P. Meade and M. Reece, hep-ph/0703031

[43] A. Djouadi, Phys. Rept. 457, 1 (2008).

[44] S. Alioli, P. Nason, C. Oleari and E. Re, JHEP 0904, 002 (2009) and JHEP 1006, 043 (2010).

[45] J. Alcaraz et al. [ALEPH and DELPHI and L3 and OPAL and LEP Electroweak Working Group Collaborations], hep-ex/0612034.

[46] M. Baak, M. Goebel, J. Haller, A. Hoecker, D. Kennedy, R. Kogler, K. Moenig and M. Schott et al., Eur. Phys. J. C 72 (2012) 2205. 